\title{
Queue Length Estimation from Probe Vehicles at Isolated Intersections: Estimators for Primary Parameters
}

\author{
Gurcan Comert \\ Department of Physics and Engineering, Benedict College, 1600 Harden St., Columbia, SC 29204 USA
}

\begin{abstract}
This paper develops estimators for market penetration level and arrival rate in finding queue lengths from probe vehicles at isolated traffic intersections. Closed-form analytical expressions for expectations and variances of these estimators are formulated. Derived estimators are compared based on squared error losses. Effect of number of cycles (i.e., short-term and long-term performances), estimation at low penetration rates, and impact of combinations of derived estimators on queue length problem are also addressed. Fully analytical formulas with unknown parameters are derived to evaluate how queue length estimation errors change with respect to percent of probe vehicles in the traffic stream. Developed models can be used for the real-time cycle-to-cycle estimation of the queue lengths by inputting some of the fundamental information that probe vehicles provide (e.g., location, time, and count). Models are evaluated using VISSIM microscopic simulations with different arrival patterns. Numerical experiments show that the developed estimators are able to point the true arrival rate values at $5 \%$ probe penetration level with 10 cycles of data. For low penetrations such as $0.1 \%$, large number of cycles of data is required by arrival rate estimators which are essential for overflow queue and volume-to-capacity ratios. Queue length estimation with tested parameter estimators is able to provide cycle-to-cycle errors within $\pm 5 \%$ of coefficient of variations with less than 5 cycles of probe data at $0.1 \%$ penetration for all arrival rates used.
\end{abstract}

Keywords: point estimation, probe vehicles, traffic signals, queue length, maximum likelihood

\section{Problem Definition}

Queue length $(\mathrm{QL})$ is an important measure for the performance of signalized intersections which can be used to estimate delays and travel times (Gartner et al. (2002); Mirchandani and Zou (2007); Lee et al. (2015); Feng et al. (2015)). The models based on these traffic parameters can be utilized for control, design, and planning purposes where the accuracy of such models becomes important. This study focuses on the estimation of low level-primary parameters for queue length models from probe vehicles (PVs) (i.e.,

\footnotetext{
${ }^{1} \mathrm{Ph}:+1(803) 705-4803$, E-mail: comertg@benedict.edu 
vehicles equipped with tracking technologies). Simple analytical closed-form expressions are developed for parameter estimators and queue length estimations (QLE) by deriving probability distributions of basic PV information types (e.g., count, location, and time). Cycle-to-cycle and steady-state behavior of parameter estimators, QLs, and their errors are formulated based on the works of Akçelik (1980) and Viti (2006). The models are evaluated with a microscopic simulation environment-VISSIM. Additional results are presented to the author's previous work. In these studies, QL estimators are developed using location and time information with known parameters (Comert and Cetin (2009, 2011); Comert (2013b)) and the effect of incorporating stop-line detection into the estimations is formulated (Comert (2013a)). This research specifically provides ability to estimate real-time queue lengths at isolated intersections, intersections with moderate to low volume-to-capacity ratios, or wherever Poisson arrivals can be assumed. Several estimators for arrival rate $\lambda$ and market penetrate rate $p$ parameters are derived that only assume simple information from probe vehicles. Single cycle and multi-cycle performance of the estimators are also presented. The study gives detailed insights about the distributions of PV information types that can be utilized for more complex models (e.g., multilane intersections) and estimating delays for signal control parameters. The results can also possibly be used as input for queue lengths/delay approximations in new signal control strategies (Comert et al. (2009); Smith et al. (2010); Goodall et al. (2013)).

The ultimate research problem investigated in this study can simply be explained as estimating the total queue length $N$ given the information from PVs (i.e., the number of probes in the queue, locations, and queue joining time instants). The estimation error of this conditional expectation from the actual $N$ is derived. For a basic illustration, Fig. 1 shows a snapshot of a signalized intersection approach at the end of red phase $(R)$ in any given cycle $(C)$. Solid cars in the figure represent PVs. For simplicity, locations of vehicles in the queue are measured in terms of number of vehicles (i.e., order of a vehicle in the queue).

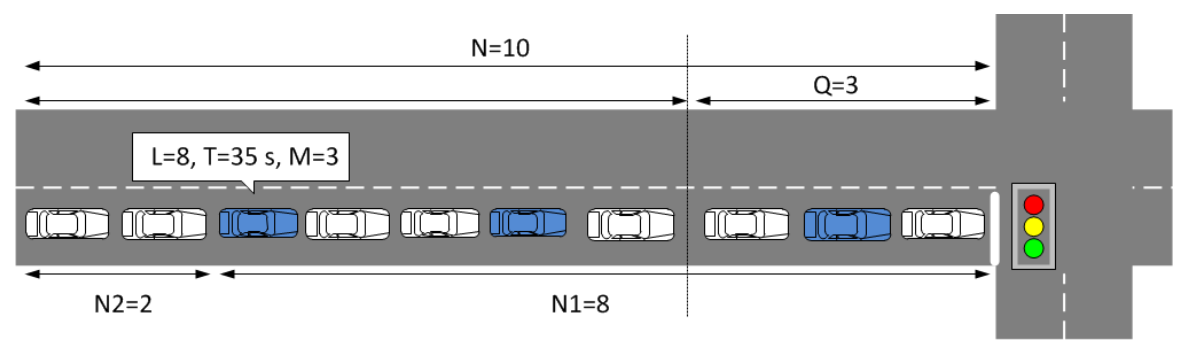

Fig. 1. Snapshot of an intersection right before the red interval terminates

Despite the fact that the fixed time signal phase lengths are used in the examples for the models, they can be applied to varying phase durations. From the practical view, developed estimators can be used for fixed or variable red phase lengths or any time interval that contains stopped probe vehicles as the PV 
data can be obtained with a snapshot of the intersection at the end of red or cycle duration. Therefore, $R$ in the formulas can be replaced by the time of estimation synced by the signal. Developed models can also be used in multilane settings given the ability of which lane level detection for probe vehicles. Then, utilizing them for finding maximum queue lengths, determining the signal timing, average overall queue length, and delay. The following notation is used throughout the paper:

$R$ and $C \quad$ red and cycle durations respectively.

$M \quad$ number of PVs in the queue (e.g., 3 in Fig. 1).

$L \quad$ the location (or the spot) of the last PV (e.g., 8) in order of vehicles from stop-bar.

$T \quad$ the time instant at which the last PV joins the back of the queue (e.g., 35 seconds that is $8^{\text {th }}$ vehicle (the last probe) joins the back of the queue 35 seconds after the red duration begins timing).

$T^{\prime} \quad$ the queue joining time of the last $\mathrm{PV}$ in the overflow queue.

$N \quad$ the total QL that is estimated in real-time at the end of red phase based on PV information.

$Q \quad$ the overflow queue which cycle-to-cycle overflows, i.e., queue at the beginning of red duration

$A \quad$ queue from the new red duration arrivals

$N_{1} \quad$ queue until the location of the last probe vehicle (up to $L=l$ )

$N_{2} \quad$ queue after the location of the last probe vehicle (between $R$ and $T$ )

$D \quad$ the difference between the actual queue length and the estimated queue length

$d(P) \quad$ the decision function or estimator of a parameter

$\lambda \quad$ arrival rate in unit vehicle per second (vps).

$p \quad$ probe proportion $\operatorname{level}(0 \approx p \leq 1), \theta=(1-p) \lambda$ is arrival rate of nonprobe vehicles which is used for simplicity in the derivations

$X \quad$ the capacity, e.g., 24 vehicles per cycle

$\rho \quad$ volume-to-capacity ratio $0<\rho \approx 1, \frac{\lambda C}{X}$

$C V \quad$ coefficient of variation, $\sqrt{V(D)} / E(N)$

For simple analytical models, the point queue model is assumed where waiting lane has infinite capacity and vehicles can accelerate and decelerate instantaneously. However, all developed models are evaluated using VISSIM which provides microsimulations with realistic vehicle movements and queuing dynamics. In addition, as described in Appendix E, different lengths of intersection approaches and random seeds facilitate evaluation of developed models under close to real data processing scenarios and arrival patterns. VISSIM estimation errors are calculated by recording vehicle data from the simulation 
and processing them in Excel Visual Basic for Applications (VBA). Apart from obvious horizontal versus vertical queuing, some discrepancies are expected among VISSIM, point queue simulations in $\mathrm{C}++$, and analytical evaluations due to:

(i) The car following in VISSIM, for a long approach lane vehicles tend to form bunches as they move downstream which makes the arrival profile not exactly Poisson (Viti (2006)).

(ii) The arrival pattern difference can also be viewed as an advantage since VISSIM evaluations show under which scenarios analytical models are accurate since platoon arrivals are expected at closely spaced intersections.

(iii) The number of runs in VISSIM are relatively low to express true steady-state behavior.

(iv) In queue definition is not optimized here. Default values are used in VISSIM (see Appendix E).

(v) VISSIM estimations use discrete time values in seconds for $T$ where analytical formula takes continuous time random variables.

(vi) VISSIM simulations involve the information process of identifying probe vehicles, their identities, and tracking them until discharged from the intersection similar to Hao et al. (2014).

Other assumptions in the paper include that the arrivals follow a Poisson distribution, vehicles accumulate in a vertical (point) queue, and PVs are able to accurately provide their locations and queue joining time information (timestamps). Poisson distribution is commonly utilized to describe arrivals at isolated intersections and the vehicles accumulate in a vertical (point) queue (Kang (2000); Mirchandani and Zou (2007); Zheng (2011); Boon et al. (2012); Lu and Yang (2014)). Limitations for Poisson models are discussed in these references. In summary, it is stated that the arrivals are correctly modeled to an isolated intersection for low volume-to-capacity ratios typically $\rho \leq 0.70$ for single channel traffic (Newell (1982)). For two or more lanes with allowed overtaking, Poisson arrivals can also be considered as an arrival process (Buckley (1962)). Furthermore, theoretical attractiveness of the Poisson arrival assumption is discussed in other fields (e.g., Paxson and Floyd (1995)) where relatively lower demand processes of connection arrivals and user generated File Transfer Protocols (FTP) are shown to be well-modeled by Poisson distribution. In another computer network modeling application, Poisson arrivals of packets to nodes are found to be suitable (Garetto and Chiasserini (2005); Cantieni et al. (2005)). Likewise, the results presented here are also applicable to those cases where vehicle arrivals can be described by a Poisson model. The assumption certainly limits the applicability. However, the study aims to show closed-form equations. Derived models can be used as simple decision tools under given assumptions. They can also be used as references in the formulations for complex intersections.

Even though locating vehicles on a link is assumed to be satisfied possibly with differential GPS (DGPS) systems (Du and Barth (2008); Herrera and Bayen (2010); Qing et al. (2011); Patire et al. 
(2015)), current accuracy may not be sufficient for field implementations especially in which lane level. Suppose that the position and the time of a vehicle waiting on the link are accurately known, the number of vehicles ahead of the observed vehicle can be estimated by an average length/spacing per vehicle. The concept of using average or effective vehicle length is common in estimating density and speed based on occupancy (i.e., percentage of time the detector is occupied/activated) measured by inductive loop detectors (e.g., Dailey (1999); Hellinga (2002); Coifman and Kim (2009); Li (2010)). Although identical type is assumed, multiple vehicle classes can be incorporated to the models by selecting the average vehicle length to be representative of different vehicle types. Multiple vehicle classes could also be added through specifying arrival rates for each vehicle type. Most certainly, processing the probe data in real life implementations can get very complex. Determination of whether a PV is in the queue or not (i.e., waiting or moving) is partially dealt with in VISSIM simulations (see Appendix E). This is usually decided by vehicle speed, acceleration and selected thresholds (e.g., Quiroga and Bullock (1998)). The paper also does not discuss information flow architecture and the exact nature of data processing needed to obtain the location or the time of PVs in the queue. However, with connected vehicle testbeds, there is a growing interest on addressing specific protocols, secure vehicle identification, the packet drop rates, effective market penetration rates, and signal fading effects through micro and macro vehicle communication network simulations (Su et al. (2014)). These factors as well as latency and high accuracy are especially important for safety critical applications.

\subsection{Literature Review}

There are several kinds of basic information that can be obtained from PVs such as speed, delay, location, time, and presence. Throughout this work, location of probes in the queue in relation to the stop bar, time instant they join the back of the queue, and total number of PVs in the queue are utilized. These types of data elements seem to be useful in estimating the arrival rate for each approach or movement at an intersection. However, the fundamental question is to determine what information to use and how to use them such that the parameters at a particular time of the day are estimated with the least error or maximum accuracy. Presented models can be used as a tool to test the optimality of the signal timing from PV data for online/offline applications. A methodology based on statistical decision theory is employed to determine what information element(s) to use and how to use them such that the arrival rate and probe proportions are estimated with the least error. Six and five estimators for the arrival rate and probe penetration levels are compared respectively according to their squared error risk values for various true $\lambda$ and $p$ levels for cycle-to-cycle and multiple cycles of data.

With field studies on connected vehicle testbeds, there is a growing body of literature that involve PV data to estimate travel times, traffic speeds, flow, and density (Hellinga and Fu (1999); Fu and Hellinga 
(2000); Kwella and Lehmann (2000); Chen and Chien (2000); Cheu et al. (2002); Cetin et al. (2005); Lin et al. (2008); Sohn and Hwang (2008); Liu and Ma (2009); Kianfar and Edara (2010); Calabrese et al. (2011); Gao and Liu (2013); Feng et al. (2014); Seo et al. (2015)). These traffic parameters are rather direct from PVs compared to delays and QLs which require statistical inference. In addition, PVs are mobile surveillance technology where the network coverage becomes an important issue. It is addressed by some studies (Boyce et al. (1991); Turner and Holdener (1995); Srinivasan and Jovanis (1996); Dion et al. (2011)). In general, the complexity of the problem makes it a challenge to develop analytical models or closed-form solutions that relate the number of probes to the accuracy of the estimates (Ferman et al. (2005); Herrera and Bayen (2010); Ou et al. (2011); Hofleitner et al. (2012); Ramezani and Geroliminis (2012); Jenelius and Koutsopoulos (2013); Tiaprasert et al. (2015)). Instead, empirical analysis or markov chain formulae are generated in these studies based on the data for numerous scenarios with different PV percentages. Typically, data from traffic simulations or sample vehicle trajectories (e.g., NGSIM) are used since real-world data with a large number of probes to support detailed analysis or specific data needs for a certain implementation are not yet available. Also analytically, shockwave-based models are developed which are mostly deterministic with a few stochastic exceptions (Ban et al. (2011); Cheng et al. (2011); Anderson et al. (2014); Jordan and Cetin (2014); Ramezani and Geroliminis (2015); Hao et al. (2013); Sun and Ban (2013); Hao et al. (2014)). Similar to this study, Hao et al. (2013) in fact give an arrival rate estimator based on last probe vehicle and signal parameters and briefly discuss the estimation of probe penetration rate. According to their numerical examples, after $10 \%$ market penetration, the estimators are able to point the exact parameter values tested. In this paper differently, multiple arrival rate and proportion estimators are derived through developed probe information distributions. Estimators are compared based on their squared error losses and accuracies in real time queue length estimations. The idea of using partial observations from actual objects to estimate system performance measures is also used in computer communication networks. In these applications, probe packets are sent from a source to one or more receiver nodes in the network in order to deduce the quality of service or performance (e.g., loss rate, delay) at the internal nodes or links (e.g., routers). In this method, performance of the internal links/nodes is estimated by exploiting the correlation present in end-to-end (origin to destination) measurements obtained from probe packets (Bowei et al. (2006); Duffield (2006)). Network, nodes, message or packet queues, and delays are also very close to the traffic applications. Specifically, communication protocols, packet drop rates, fading, and information processing for vehicle probes are investigated (Hartenstein and Laberteaux (2008); Park et al. (2011)).

Early studies in traffic signal queues focus on simulation analysis, curve fitting, and efforts to formulate expected value and variance of average QL for different arrival distributions (Adams (1936); Webster 
(1958); Haight (1959); Miller (1963); Darroch (1964); Newell (1965); McNeil (1968); Heidemann (1994)). Furthermore, formulations are given without and with the overflow queue (the leftover queue from a previous cycle) and approximations to average overflow queue (Ohno (1978); Tarko and Rouphail (1994); Van Den Broek et al. (2006)). Later on, formulas are derived for the steady-state probability generating function of the overflow queue, numerical evaluations of parameters (Van Leeuwaarden (2006)), and Markov Chain formulations of the overflow queue and the total QL (Olszewski (1990); Van Leeuwaarden (2006); Viti and Van Zuylen (2010)). In the literature, several models exist for the expected value of overflow queue and relatively fewer models can be found for the variance (Medhi (1991); Fu and Hellinga (2000); Viti and Van Zuylen (2010)). Cycle-to-cycle behavior of expected queue lengths and variances are derived in Rouphail et al. (2001); Viti (2006).

The rest of the paper is organized as follows. Problem statement and notations are introduced in Section 1. Section 2 describes analytical formulations of the probability distributions and expected values of parameter estimators for the PV information types. Section 3 presents derivations for the QLE problem without and with the overflow queue cases as well as approximations to the overflow queue case. Numerical examples are given for each case along with VISSIM comparisons. Lastly, Section 4 summarizes the findings. Detailed step-by-step derivations are also provided in the appendix.

\section{Derivation of Parameter Estimators}

In this section, estimators for $\lambda$ and $p$ are presented. Expected values and estimation errors are derived using probability distributions of the PV information in the queue. Suppose that every vehicle has equal probability of being a PV (i.e., $P\left(y_{j}=1\right)=p$ ) and arrival distributions of probes and nonprobes are identical with different parameters (i.e., $p \lambda$ and $\theta=(1-p) \lambda$ respectively). This may not be always true (Hellinga and $\mathrm{Fu}(2002)$ ). Suppose that random variables $y_{1}, y_{2}, \ldots$, where $y_{j}$ denotes the $j^{\text {th }}$ vehicle in the queue. And, assume that they are independent of each other and independent of the total number of all vehicles in the queue $N$. The relationship between the total number of probe vehicles $M$ and the total queue length $N$ can be written as a compound random variable $M=\sum_{j=1}^{N} y_{j}$ where $y_{j} \in 0,1$ and $P\left(y_{j}=1\right)=p$.

Clearly, both $N$ and $M$ are discrete random variables. $M$ denotes the number of independent PV arrivals within total of $N=n$ arrivals. Probability of the number of PVs in the queue $(M=m \mid N=n)$ follows a Binomial $(n, p)$. Under the assumption of Poisson arrivals with mean $\lambda R$, probability mass function (pmf) of the number of probes in the queue $M$ at the end of red phase of a traffic light, pmf of location of the last probe $L$, the probability density function (pdf) of queue joining time of the last probe can be given as in Eqs. (1)-(3). Detailed of derivations of these marginal distributions can be found in 
Comert (2013b).

$$
\begin{gathered}
P(M=m)=\frac{e^{-p \lambda R}(\lambda p R)^{m}}{m !}, \text { for } 0 \leq p \leq 1, m=0,1,2, \ldots \\
P(L=l)= \begin{cases}\frac{p}{(1-p)^{l}} \sum_{n=l}^{\infty} \frac{e^{-\lambda R}(\theta R)^{n}}{n !}, & l \geq 1 \\
\sum_{n=0}^{\infty} \frac{e^{-\lambda R}(\theta R)^{n}}{n !}=e^{-p \lambda R}, & l=0\end{cases} \\
f(t)= \begin{cases}\frac{\lambda p e^{-p \lambda(R-t)}}{\left(1-e^{-\lambda p R}\right)}, t<R \\
0, \text { o.w. }\end{cases}
\end{gathered}
$$

In order to derive parameter estimators, one can use joint pdfs of PV information. These distributions can be found as in Eqs. (4) and (5) after utilizing conditional distributions $f(t, l)=f(t \mid l) P(L=l)$ as presented in Appendix A.

$$
f(t, l)=\frac{(\lambda p)(\lambda t)^{l-1} e^{-t \theta} e^{-p \lambda R}}{(l-1) !}, L>0
$$

The joint probability density function of $f(t, l, m)$ is obtained by $f(t, l, m)=f(t \mid l, m) P(L=l, M=m)$. After simplifications $f(t, l, m)$ can be written as,

$$
f(t, l, m)=\left\{\begin{array}{l}
\frac{p^{m}(1-p)^{-m} \theta(\theta t)^{l-1} e^{-t \theta} e^{-p \lambda R}}{(m-1) !(l-m) !}, M>0 \\
e^{-p \lambda R}, M=0
\end{array}\right.
$$

In the next step using Eqs. (4) and (5), maximum likelihood estimators (mle) of $\lambda$ and $p$ can be deduced. Table 1 shows the complete list of estimators studied in this paper. Step-by-step derivations of these estimators are given in Appendix A. Total of six alternatives are given for $\lambda$ and five are given for $p$. It is possible to come up with additional estimators, but, these seem to be simple choices to consider. Simple assumptions for each estimator can be introduced as:

Table 1. Estimators for $p$ and $\lambda$

\begin{tabular}{lccc}
\hline Information & \multicolumn{1}{c}{$\hat{p}$} & Information & $\hat{\lambda}$ \\
\hline$M$ & $\hat{p}_{1}=m /(\lambda R)$ & $M$ & $\hat{\lambda}_{1}=m / p R$ \\
$M, L$ & $\hat{p}_{2}=m / l$ & $L$ & $\hat{\lambda}_{2}=l / R$ \\
$T$ & $\hat{p}_{3}=1 /[\lambda(R-t)]$ & $L, T$ & $\hat{\lambda}_{3}=l / t$ \\
$L, T$ & $\hat{p}_{4}=t /[(R-t)(l-1)]$ & $L, T$ & $\hat{\lambda}_{4}=(l-1) / t$ \\
$L, T, M$ & $\hat{p}_{5}=m t /(m t+(l-m) R)$ & $L, T$ & $\hat{\lambda}_{5}=l /(t+p(R-t))$ \\
& & $L, T, M$ & $\hat{\lambda}_{6}=(l-m) / t+m / R$ \\
\hline
\end{tabular}

P1. $\hat{p}_{1}=m /(\lambda R)$ is directly derived by assuming $M=m$ number of PVs and $\lambda$ are assumed to be known.

It is mle of $p$ from the distribution of number of probe vehicles in Eq. (1).

P2. $\hat{p}_{2}=m / l$ uses the information of $L=l, M=m$ and the assumption of $P(l \mid m)=\left(\begin{array}{c}l-1 \\ m-1\end{array}\right) p^{m-1}(1-p)^{l-m}$. Then, $P(l, m)$ can be written as $P(l, m)=\left(\begin{array}{c}l-1 \\ m-1\end{array}\right) p^{m-1}(1-p)^{l-m} \frac{(\lambda p R)^{m} e^{-\lambda p R}}{(m) !}$ and the mle of $p$ is $m / l$. 
P3. $\hat{p}_{3}=1 /[\lambda(R-t)]$ needs location and time information. It is mle of $p$ from $f(t, l)$ in Eq.(4).

P4. $\hat{p}_{4}=t /[(R-t)(l-1)]$ uses location and time information. It is obtained by plugging $\hat{\lambda}_{4}$ in $\hat{p}_{3}$.

P5. $\hat{p}_{5}=m t /(R(l-m)+m t)$ utilizes $L, T, M$ information, the estimator is derived by finding the mle of $p$ using Eq.(5).

L1. $\hat{\lambda}_{1}=m /(p R)$ is directly obtained by finding mle of $\lambda$ from in Eq.(1). It assumes $M=m$ and $p$ are known.

L2. $\hat{\lambda}_{2}=l / R$ utilizes only $L=l$ information. It is derived by plugging $\hat{p}_{2}$ in $\hat{\lambda}_{1}$ as $\frac{m}{\hat{p}_{2} R}=\frac{m}{m R / l}=\frac{l}{R}$.

L3. $\hat{\lambda}_{3}=l / t$ is deduced by observation of $L=l$ number of vehicles arrive within $t$ seconds after the light turns red.

L4. $\hat{\lambda}_{4}=(l-1) / t$ is using $L, T$ and obtained by plugging $\hat{p}_{3}=1 /[\lambda(R-t)]$ in $\hat{\lambda}_{5}$.

L5. $\hat{\lambda}_{5}=l /[t+p(R-t)]$ utilizes location and time information in the joint distribution of $L, T$. It is mle estimator of $\lambda$.

L6. $\hat{\lambda}_{6}=[(l-m) / t]+(m / R)$ allocates two or more information types with no known parameter assumption. Using $L, T, M$, the estimator is derived from $f(t, l, m)$ in Eq. (5). It is mle for $\lambda$.

Estimators are direct mle of parameters under the assumptions of either none or one parameter is known as presented in Table 1. In practice, as the simplest information type-count from PVs $M$ can be obtained and others (i.e., location or time) may not be available. This may be observed in traffic data such as speed may be not available when count data is obtained from loop detectors. Clearly, count information in general is rather direct compared to speed or density which require inference like average vehicle sizes and effective detector lengths etc. Similarly for PVs, location and time information involve inference due to positioning and time referencing. Therefore, which type of information can be used for what accuracy level are interesting research questions. Certainly, with only PV counts in the queue either $\lambda$ or $p$ assumed to be known (e.g., stop-bar detector or combination of other technology). Alternatively, there may be at least a prior knowledge in case of multi-cycle applications as these parameters can change over time. Hence, initial knowledge can evolve overtime.

\subsection{Expectations}

In order to compare the proposed estimators and identify better candidates under different scenarios, behavior of steady-state and cycle-to-cycle expected values can be checked first. Even though, overflow queue is assumed to be zero $Q=0$ for closed-formulas, the effect of $Q>0$ is shown via simulations and approximations. Use of $Q=0$ can enable complete closed form formulations, simple evaluations, and until what volume-to-capacity ratios can derived QL estimators be utilized. Expected value of $\hat{\lambda}_{1}$ and $\hat{p}_{1}$ as $E\left(\hat{\lambda}_{1}\right)=E(m / p R)=\lambda p R / p R=\lambda$ and $E\left(\hat{p}_{1}\right)=E(m /(\lambda R))=\lambda p R / \lambda R=p$ are straightforward. Analytical 
formulations and approximations are shown in Table 2 and detailed derivations are provided in Eqs. (B.1)(B.15). These estimators can be used without and with overflow queue cases as long as a probe is present in the current cycle. These information can be inferred from vehicle index number or delay experienced. Clearly, values are not expected to be exact. Particularly in VISSIM evaluations some inference is devoted to obtain the useful information such as location $L=l$ which includes the vehicles in overflow queue:

1. When $Q>0,\left(L=l \mid l_{i-1} \in Q\right)$ slightly overestimates and $\left(L=l \mid l_{i-1}=0\right)$ would be largely overestimating the true location of the last probe vehicle.

2. For $Q=0, L=l$ can show the true location.

3. $M=m$ can be obtained given that there is at least one PV in the current cycle arrivals. From temporary vehicle numbers it can be inferred. How long these numbers can be kept is a design problem.

4. Given there is at least one PV in the queue, $T$ is also direct information. Simply, vehicles may have time counters for a specific intersection.

In addition, free flow PVs are partly addressed like real life cases in VISSIM where detection zones are selected and the information from stopped/near stopped PVs are counted. So VISSIM evaluations include those scenarios. However, green arrivals are ignored which could also be incorporated by already discharged and just joining PVs. Hence, models here are not estimating the maximum cycle-to-cycle QLs. They are rather cycle-to-cycle red duration QLs which can be used for next cycle green timing. Incorporation of the handling of probe information, therefore, is partly addressed in point queue and VISSIM simulations. Numerical results are obtained including the information errors that can be possibly experienced in real life applications. Analytically traceable simple formulas for the estimators are given when overflow queue is ignored. When overflow queue is included, simulations from point queue and VISSIM are provided to compare the behaviors. Expected values and errors closely follow true values as arrival rate and probe proportions are estimated with new arrivals when at least one PV exists in the queue. Analytical formulas provide the steady-state behavior of the estimators. Point queue simulations are close to them, but, VISSIM results are different as number of cycles are relatively lower. In addition, analytical formulas are derived for single-cycle (real-time) applications. Multi-cycle results that are similar to rolling horizon approach are also provided using point queue simulations. Fig. 2 shows steady-state expected values of $\hat{\lambda}_{\mathrm{s}}$ which are calculated by analytical formulas, point queue simulations, and VISSIM evaluations. Analytical results demonstrate single-cycle steady-state values for $Q=0$. Simulations give weighted evaluations with 5\% new cycle and $95 \%$ historical averages over high number of cycles when $Q \geq 0$. This percentage can be optimized, however, it is chosen based on good performance after number of numerical evaluations. Apart from the differences discussed in section 1, discrepancies with analytical 
Table 2. Expected values of compared estimators

\begin{tabular}{|c|c|c|}
\hline Estimator & Expected value & Approximation \\
\hline$E\left(\hat{p}_{1}\right)=E(m /(\lambda R))$ & $p$ & - \\
\hline$E\left(\hat{p}_{2}\right)=E(m / l)$ & $p+(1-p) E(1 / l)$ & $\frac{\lambda p^{2} R\left(1-e^{-\lambda p R}\right)}{\lambda R-(1-p)\left(1-e^{-\lambda p R}\right) / p}$ \\
\hline$E\left(\hat{p}_{3}\right)=E[1 /(\lambda(R-t))]$ & $\int_{0}^{R} \sum_{l=1}^{\infty} \frac{f(t, l)}{\lambda(R-t)} d t$ & $\begin{array}{c}\lambda R-(1-p)\left(1-e^{-\lambda p R}\right) / p \\
\frac{p}{\left(1-e^{-\lambda p R}\right)}\end{array}$ \\
\hline$E\left(\hat{p}_{4}\right)=E[t /((R-t)(l-1))]$ & $\int_{0}^{R} \sum_{l=1}^{\infty} \frac{t f(t, l)}{(R-t)(l-1)} d t$ & $\frac{p}{\left(1-e^{-\lambda p R}\right)}$ \\
\hline$E\left(\hat{p}_{5}\right)=E[m t /(m t+(l-m) R)]$ & $\int_{0}^{R} \sum_{l=1}^{\infty} \sum_{m=1}^{l-1} \frac{m t f(t, l, m)}{m t+(l-m) R} d t$ & $\frac{E(m)}{E(m)+R E(l-1 / t)-R E(m-1 / t)}=\frac{\lambda p R}{\lambda p+\lambda R-\lambda p}=p$ \\
\hline$E\left(\hat{\lambda}_{1}\right)=E(m /(p R))$ & $\lambda$ & ( \\
\hline$E\left(\hat{\lambda}_{2}\right)=E(l / R)$ & $\lambda-\frac{(1-p)\left(1-e^{\lambda p R}\right)}{p R}$ & - \\
\hline$E\left(\hat{\lambda}_{3}\right)=E(l / t)$ & $\theta E\left(\frac{l}{l-1}\right)+\lambda p+\lambda p E(1 / x)$ & $\lambda+\frac{\lambda p^{2}\left(1-e^{\lambda p R}\right)}{\lambda R p-\left(1-e^{-\lambda p R}\right)}$ \\
\hline$E\left(\hat{\lambda}_{4}\right)=E((l-1) / t)$ & $\lambda$ & - \\
\hline$E\left(\hat{\lambda}_{5}\right)=E(l /((1-p) t+p R))$ & $\int_{0}^{R} \sum_{l=1}^{\infty} \frac{l f(t, l)}{(1-p) t+p R} d t$ & $\frac{\lambda R-(1-p)\left(1-e^{-\lambda p R}\right) / p}{R-(1-p)\left(1-e^{-\lambda p R}\right) / \lambda p}=\lambda$ \\
\hline$E\left(\hat{\lambda}_{6}\right)=E\left(\frac{l-m}{t}+\frac{m}{R}\right)$ & $\lambda$ & $\begin{array}{c}n-(1-p)(1-e \\
-\end{array}$ \\
\hline
\end{tabular}

evaluations are due to the approximation for overflow queue and use of single-cycle data. In analytical evaluations, it can be seen that $\hat{\lambda}_{4}$ and $\hat{\lambda}_{6}$ are unbiased. Performance of $\hat{\lambda}_{5}$ is able to point the true $\lambda$ after $p \geq 10 \%$. From simulation evaluations, steady-state figures assume fixed $p$ and $\lambda$ parameters over a longer period (e.g., $\approx 25$ hours for VISSIM simulations). In Fig. 2, it can be seen that all of the estimators show pretty good performances for high $\rho$ levels. For low arrival rates, $\hat{\lambda}_{4}, \hat{\lambda}_{5}$, and $\hat{\lambda}_{6}$ show similar behavior. Although estimator $\hat{\lambda}_{1}$ is also unbiased, $p$ is needed to be known. In real life single-cycle applications at especially low $p$ levels, this estimator can give large values due to integer $M$ values. For example when $\lambda=0.239$ vehicles per second (vps), $M=1, p=0.001, R=45, \hat{\lambda}_{1}=22.22$ vps which is highly inaccurate. This problem can be partly solved by using historical values and combining them with real-time data (i.e., using average $M \mathrm{~s}$ ). Furthermore, it can be seen that the estimator $\hat{\lambda}_{2}=l / R$ uses only $L=l$, however, it is biased. Magnitude of bias reduces as $p$ increases. From Fig. 6, it has low error. Nevertheless, it is not a good estimator to embed in QLE especially for low $p$ levels. It underestimates the arrival rate at low probe proportions leading to underestimation of overflow queue for high $\rho$ values. Given that $(L, T)$ is observed, to substitute in QLE formula $\hat{\lambda}_{4}=(l-1) / t$ is a better estimator.

Fig. 3 exhibits all types of evaluations for $\hat{p}_{2}, \hat{p}_{4}$, and $\hat{p}_{5}$. The estimator $\hat{p}_{1}$ is unbiased and able to show the true $p$ over long run. It is not shown in the figure as it assumes $\lambda$ is known and analytically $E\left(\hat{p}_{1}\right)=p$ is trivial. It can be seen that $\hat{p}_{2}$ and $\hat{p}_{5}$ could easily be used for estimating $p$. Estimators $\hat{p}_{2}$ and $\hat{p}_{4}$ are biased and $\hat{p}_{3}$ shows very similar behavior with $\hat{p}_{4}$ as $t /(l-1)$ approaches to $\lambda$. These estimators give comparable results with multiple cycles of probe data.

Under the assumptions of unchanging parameter levels, it can be interesting to find estimators that can point true values less than 30 minutes (or $\approx 20$ cycles). So, performance of the estimators are shown for short-term (e.g., $i=2-50$ cycles) using point queue simulations. In Hofleitner et al. (2012), arterial state estimation arrival and market penetration rates are assumed to be constant within 5-15 minutes. It can be reasonable to 

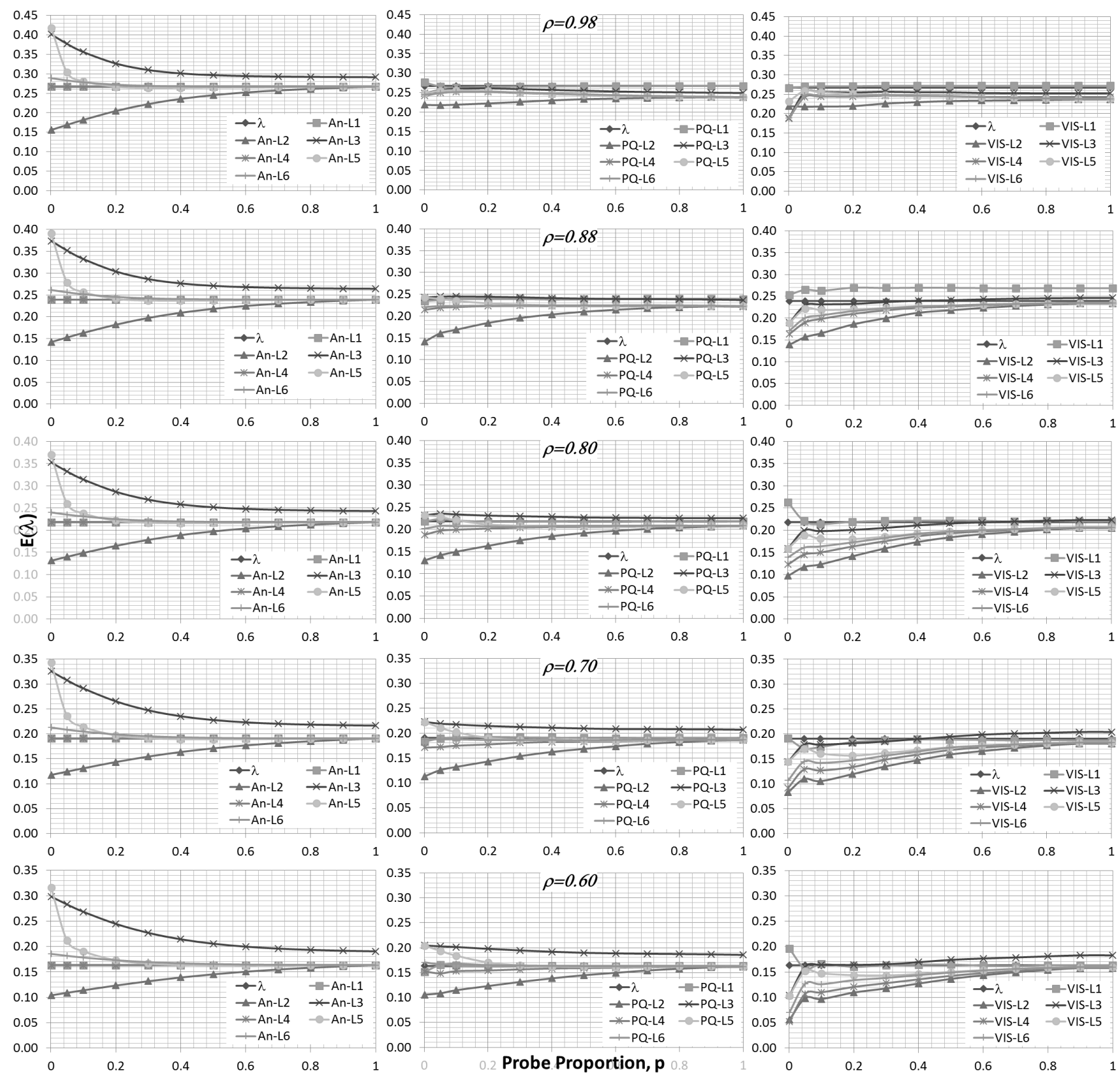

Fig. 2. Steady-state expected value of the estimators for $\lambda$ for various $\rho$ levels evaluated by analytical, point queue, and VISSIM simulations

assume unchanging parameters within 15 cycles. Figs. 4 and 5 illustrate the cycle-to-cycle behavior of expected value of the estimators up to 50 cycles (i.e., $\approx 1.25$ hours). Broadly, estimators that contain $M$ are performing better since $\sum_{k=1}^{i} \frac{M_{k}}{i}$ approaches to $\lambda p R$ as number of cycles increase. On the other hand, $L$ and $T$ are only relevant to the back of queue (i.e., they can expected to be towards the front or back) rather than number of new arrivals and cycles. Nevertheless, $\hat{\lambda}_{2}$ and $\hat{\lambda}_{3}$ are biased estimators. From Fig. 4 , when $p=0.001$, all of the $\lambda$ estimators perform poorly. For $p=0.05$, the difference from true $\lambda$ decreases to $0 \%$ at about 15 cycles except $\hat{\lambda}_{2}$. Although similar performance can be observed for 

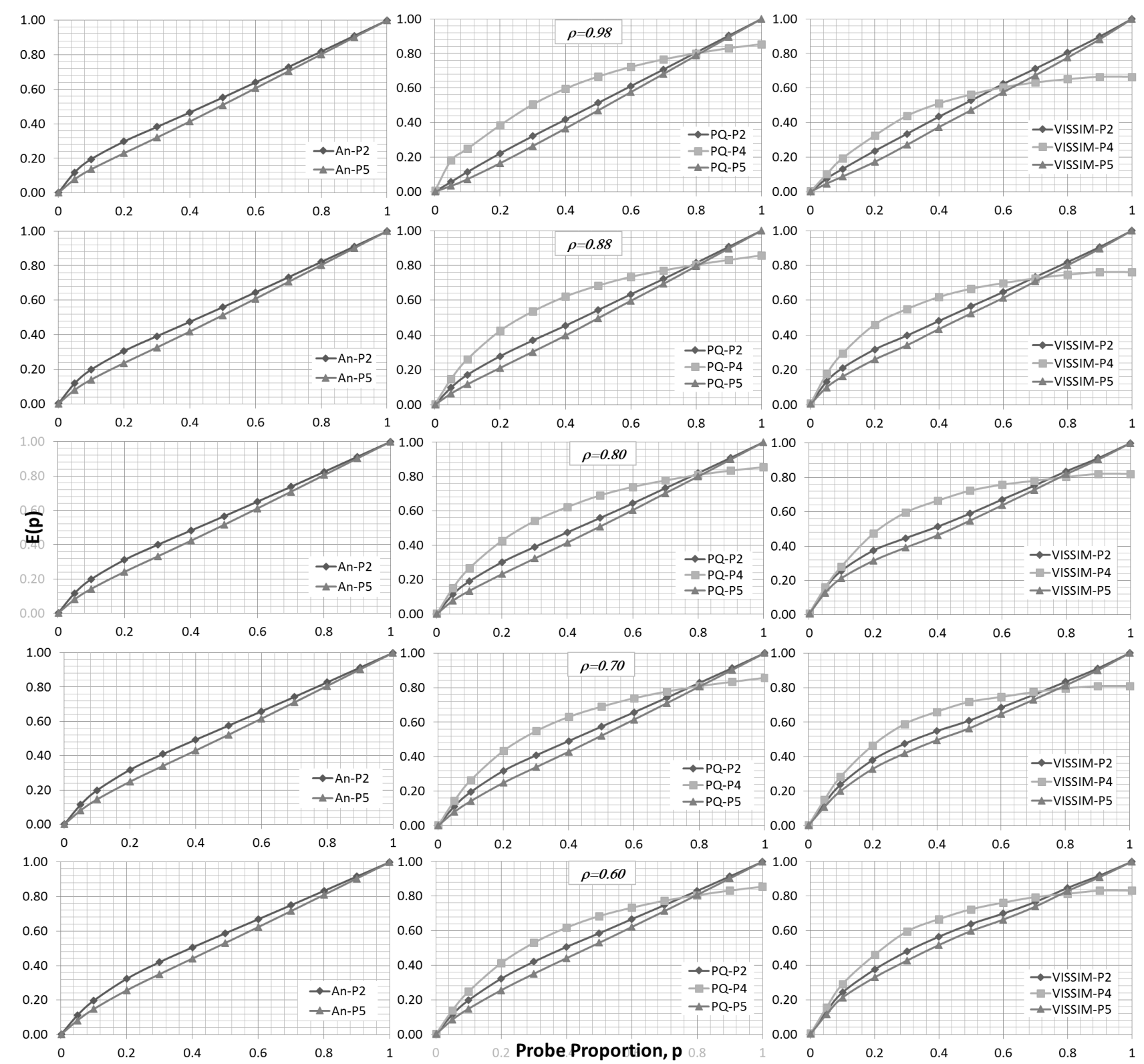

Fig. 3. Steady-state expected value of the estimators for $p$ at various $\rho$ levels evaluated by analytical, point queue, and VISSIM simulations

$\rho=0.88$ and lower $\rho$ values, the difference reduces to $0 \%$ comparably at higher number of cycles. As expected, for higher $p$ levels, accuracy of the estimators increases except again $\hat{\lambda}_{2}$ which gives the true parameter value at $p=1.00$ (see Fig. 2). $\quad$ In Fig. 5, for all $\rho$ and $p$ levels estimators, $\hat{p}_{2}$ and $\hat{p}_{5}$ are able to point the true parameter values between 5 to 10 cycles where percent differences reduce to less than $10 \%$. From the two estimators, $\hat{p}_{5}$ is better which stays within $\pm 5 \%$ of the true parameter value. It demands all three information of $(L, T, M)$ where $\hat{p}_{2}$ gives less than $\pm 10 \%$ using $(L, M)$. Moreover, estimators for $p$ are showing much better performance compared to $\hat{\lambda}$ s at all levels of $p$ and $\rho$ staying less than $\pm 0.7 \%$ for $p=0.001$ and $\pm 5 \%$ for $p=0.05$. Overall, $\hat{p}_{5}$ shows the best performance for all true 

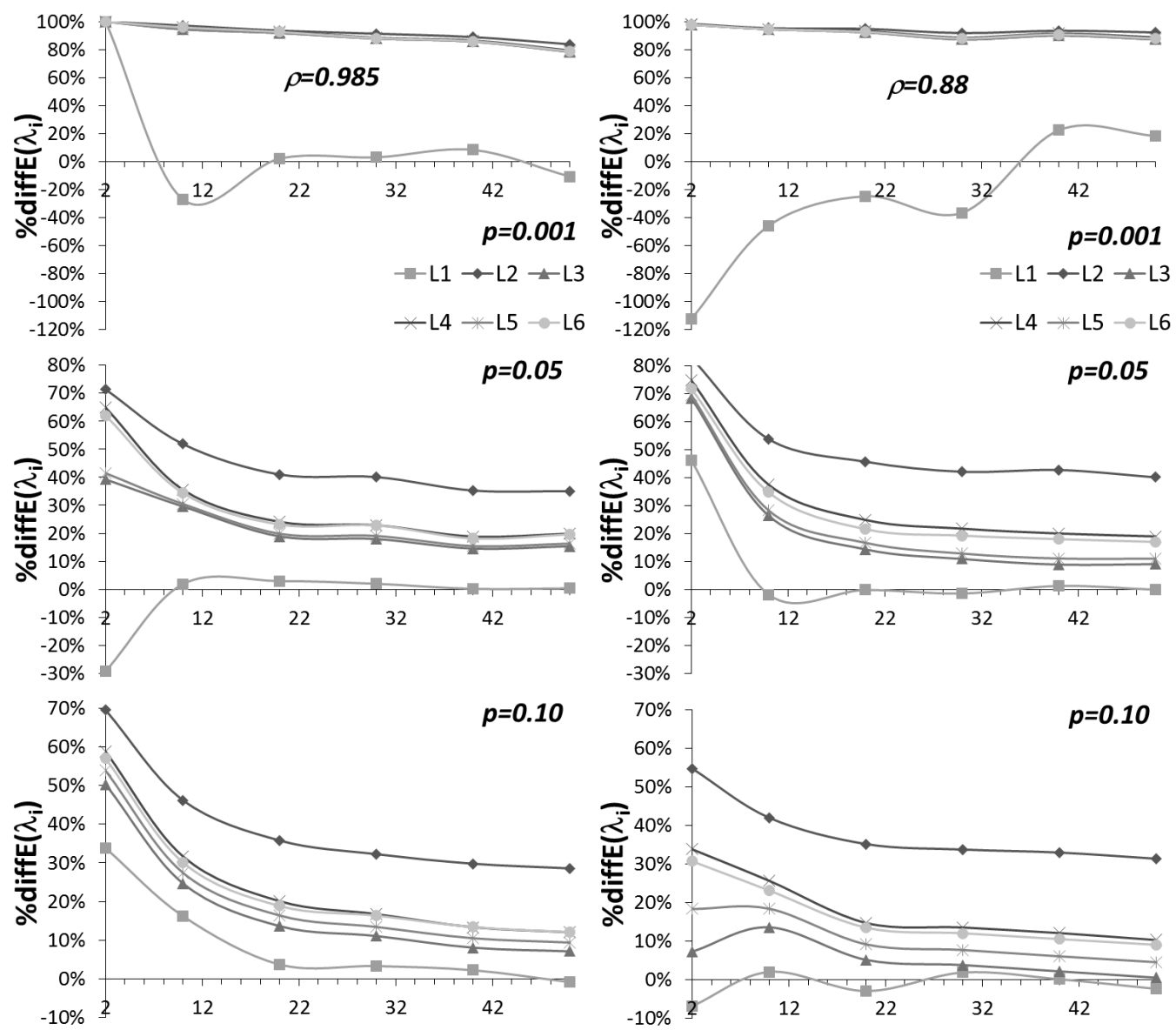

Fig. 4. Up to $i=50$ cycle-to-cycle expected value of the estimators for $\lambda$ at $\rho=0.98,0.88$ and $p=0.001,0.05,0.10$

parameter levels.

\subsection{Squared Error Losses}

Alternative estimators can also be compared with respect to their estimation errors (e.g., mean squared error losses) which are calculated as shown below for repeated data collected for single cycle. Similarly, as in the previous section, analytical estimation errors are given when possible. Furthermore, effect of multiple cycles and scenarios for different possible applications are discussed through simulation evaluations.

$$
E(d(P)-\lambda)^{2}=V(d(P))+[E(d(P))-\lambda]^{2}=V(d(P))+\left[\operatorname{Bias}_{\lambda} d(P)\right]^{2}
$$

Mean squared error for an estimator simply equals to sum of the variance of estimator and the bias given in Eq. (6) where $d(P)$ denotes the estimator function. For $\hat{\lambda}_{1}$, it can be verified that the estimator is unbiased since $E(m / p R)=\lambda$. Thus, mean squared error loss is equal to its variance and can be evaluated as $V(m / p R)=\lambda /(p R)$. All of the estimation errors by definition are given in Eqs. (C.2)(C.12). Whenever analytical derivations of mean squared errors are traceable, closed-form equations of the errors are given. For complicated overflow case, approximations, bounds, and simulation evaluations 


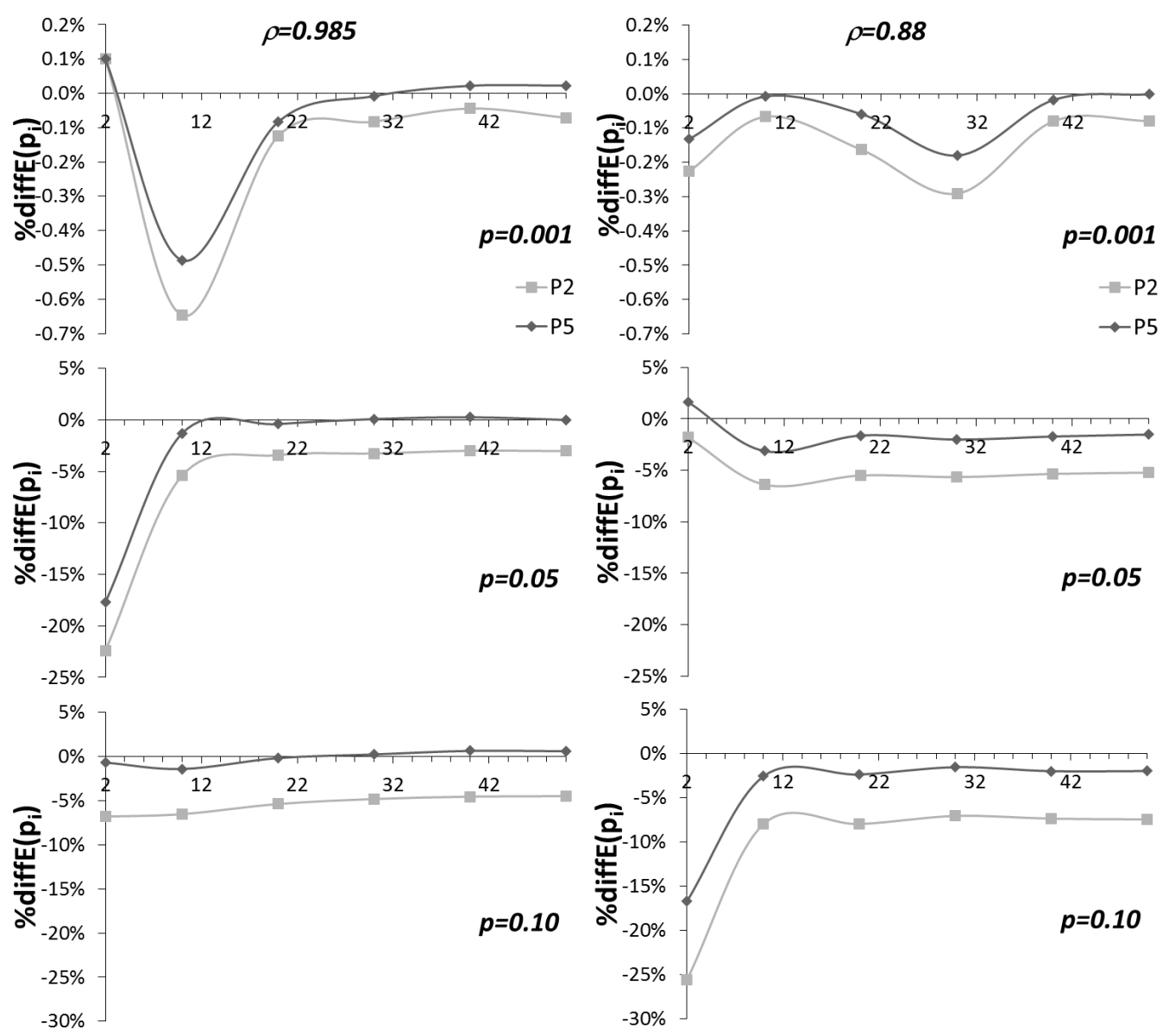

Fig. 5. Up to $i=50$ cycle-to-cycle $E\left(p_{i}\right) \mathrm{s}$ at $\rho=0.98,0.88$ and $p=0.001,0.05,0.10$

Table 3. Errors of estimators

\begin{tabular}{lcc}
\hline Error & Formula & Approx bound \\
\hline$E\left([m /(\lambda R)-p]^{2}\right)$ & $p /(\lambda R)$ & - \\
$E\left([m / l-p]^{2}\right)$ & $E\left[\frac{(l-1) p(l p-2 p+3)}{l^{2}}\right]-2 p(p+(1-p) E(1 / l))+p^{2}$ & $\frac{1}{n E\left(\left[2 m / p^{2}+\left[(l-m) /(1-p)^{2}\right]\right)\right.}$ \\
$E\left([1 /[\lambda(R-t)]-p]^{2}\right)$ & $\int_{0}^{R} \sum_{l=1}^{\infty}(1 /[\lambda(R-T)]-p)^{2} f(t, l) d t$ & $\frac{p^{2}}{n}$ \\
$E\left([t /((R-t)(l-1))-p]^{2}\right)$ & $\int_{0}^{R} \sum_{l=1}^{\infty}(t /[(l-1)(R-t)]-p)^{2} f(t, l) d t$ & $\frac{p^{2}}{n}$ \\
$E\left([(m t /(m t+(l-m) R))-p]^{2}\right)$ & $\int_{0}^{R} \sum_{l=1}^{\infty} \sum_{m=1}^{l-1}(m t /[m t+(l-m) R]-p)^{2} f(t, l, m) d t$ & $\frac{1}{n E\left(\left[m / p^{2}\right]+\left[(l-m) /(1-p)^{2}\right]\right)}$ \\
\hline$E\left([m /(p R)-\lambda]^{2}\right)$ & $\lambda /(p R)$ & - \\
$E\left([l / R-\lambda]^{2}\right)$ & $\frac{\lambda(3 p-2)}{R p}+\frac{\left(1-e^{\lambda p R}\right)\left(p^{2}-3 p+2+2 \lambda(1-p)\right)}{R^{2} p^{2}}$ & - \\
$E\left([l / t-\lambda]^{2}\right)$ & $\int_{0}^{R} \sum_{l=1}^{\infty} \frac{\left(l^{2}\right) f(t, l)}{t^{2}} d t-2 \lambda \int_{0}^{R} \sum_{1}^{\infty} \frac{(l) f(t, l)}{t} d t+\lambda^{2}$ & $\frac{\lambda^{2}}{n E(l)}$ \\
$E\left([(l-1) / t-\lambda]^{2}\right)$ & $\sum_{l=1}^{\infty} \frac{(l-1) p \theta^{2} R e^{-p \lambda R}}{(l-2)(1-p) l^{l}} \sum_{n=l-2}^{\infty} \frac{(\theta R)^{n} e^{-\theta R}}{n !}-2 \lambda\left(1-e^{-\lambda p R}\right)+\lambda^{2}$ & $\frac{\lambda^{2}}{n E(l)}$ \\
$E\left([(l /((1-p) t+p R))-\lambda]^{2}\right)$ & $\left.\int_{0}^{R} \sum_{l=1}^{\infty}(l /(t+p(R-t))]-\lambda\right)^{2} f(t, l) d t$ & $\frac{\lambda^{2}}{n E(l)}$ \\
$E\left(\left[\left(\frac{l-m}{t}+\frac{m}{R}\right)-\lambda\right]^{2}\right)$ & $\int_{0}^{R} \sum_{l=1}^{\infty} \sum_{m=1}^{l-1}([(l-m) / t+r / m]-\lambda)^{2} f(t, l, m) d t$ & $\frac{\lambda^{2}}{n E(l)}$ \\
\hline
\end{tabular}

are presented. Figs. 6 and 7 for estimation errors are obtained solely from analytical formulas for $Q=0$. When overflow queue is included analytical error bounds and simulation results from point queue and VISSIM are presented. Analytical estimation error bounds for single cycle probe information are found by utilizing the Cramér-Rao Lower Bound. Estimation error lower bounds are close to analytical errors, 
the difference is caused by truncation $l / t$ for $t>0$ in integration of summations. Under some scenarios, simulation results are lower because of weighting new and historical data from previous cycles. Point queue simulations estimate the parameters by weighting the probe data values (i.e., $95 \%$ with $5 \%$ of the current cycle data value). The historical values are averaged as they become available, thus, with $i=10^{3}$ runs sum of data values is divided to $10^{3}$ cycles so that estimators' steady-state behaviors are obtained. For QLEs with estimators $\hat{\lambda}$ and $\hat{p}$ at $i^{\text {th }}$ cycle is used and error of queue length estimations (i.e., $V(D)$ s) are reported. Figs. 6 and 7 are derived to visualize the estimators' performances based on
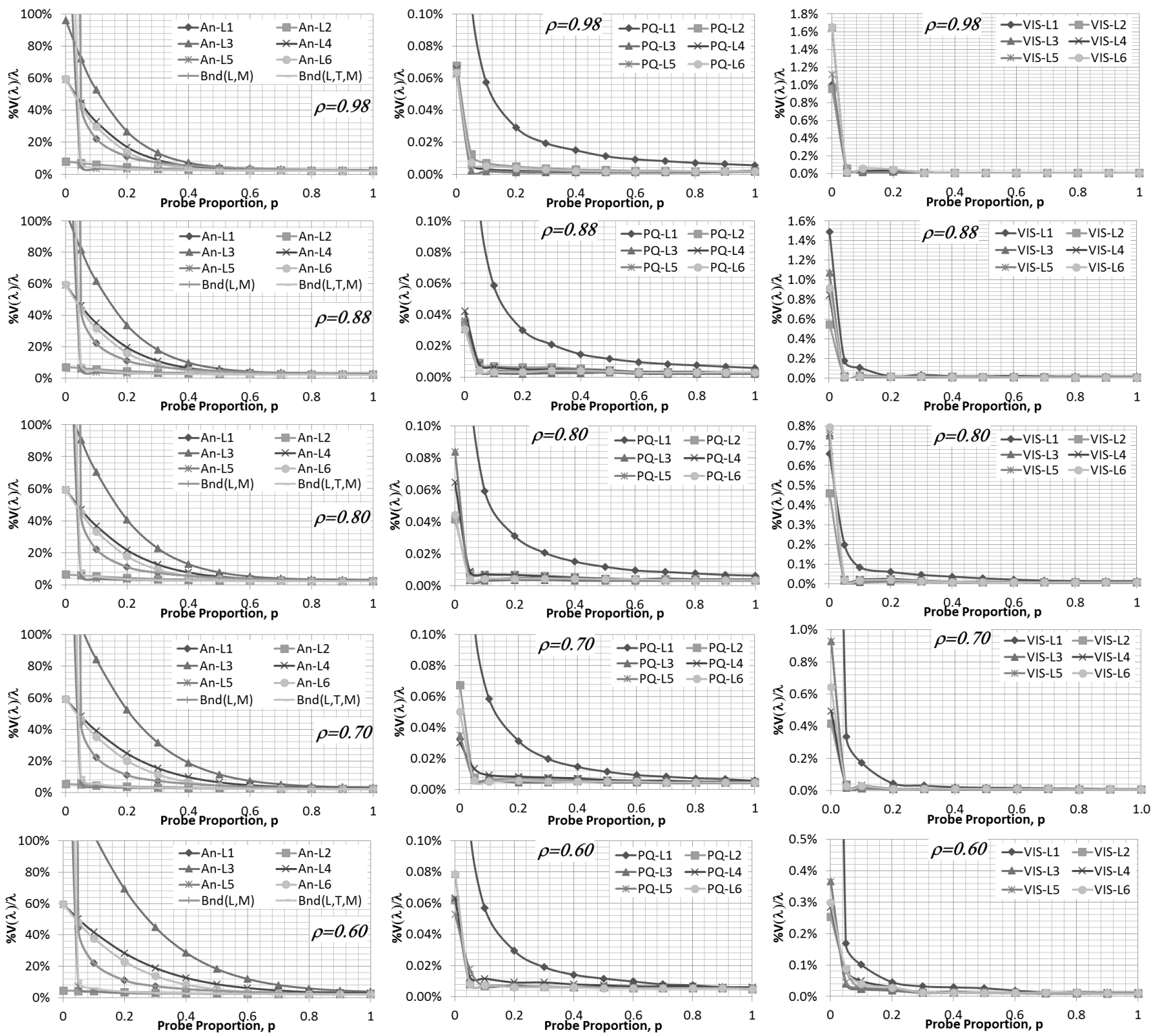

Fig. 6. Steady-state $\% V(\lambda) / \lambda$ of the estimators for $\lambda$ for various $\rho$ levels evaluated by analytical, point queue, and VISSIM simulations

relative errors $\% V(\lambda) / \lambda$ and $\sigma(\hat{p})$. Both errors are denoted in percent. In Fig. 6, estimators are able to provide less than $2 \%$ of the true arrival rate parameter at $\rho=0.98$ and errors reduce to less than $0.5 \%$ 
level as $\rho$ decreases. Similarly from Fig. 7, standard deviation is about $10 \%$ for $\hat{p}_{2}$ and less than $5 \%$ for
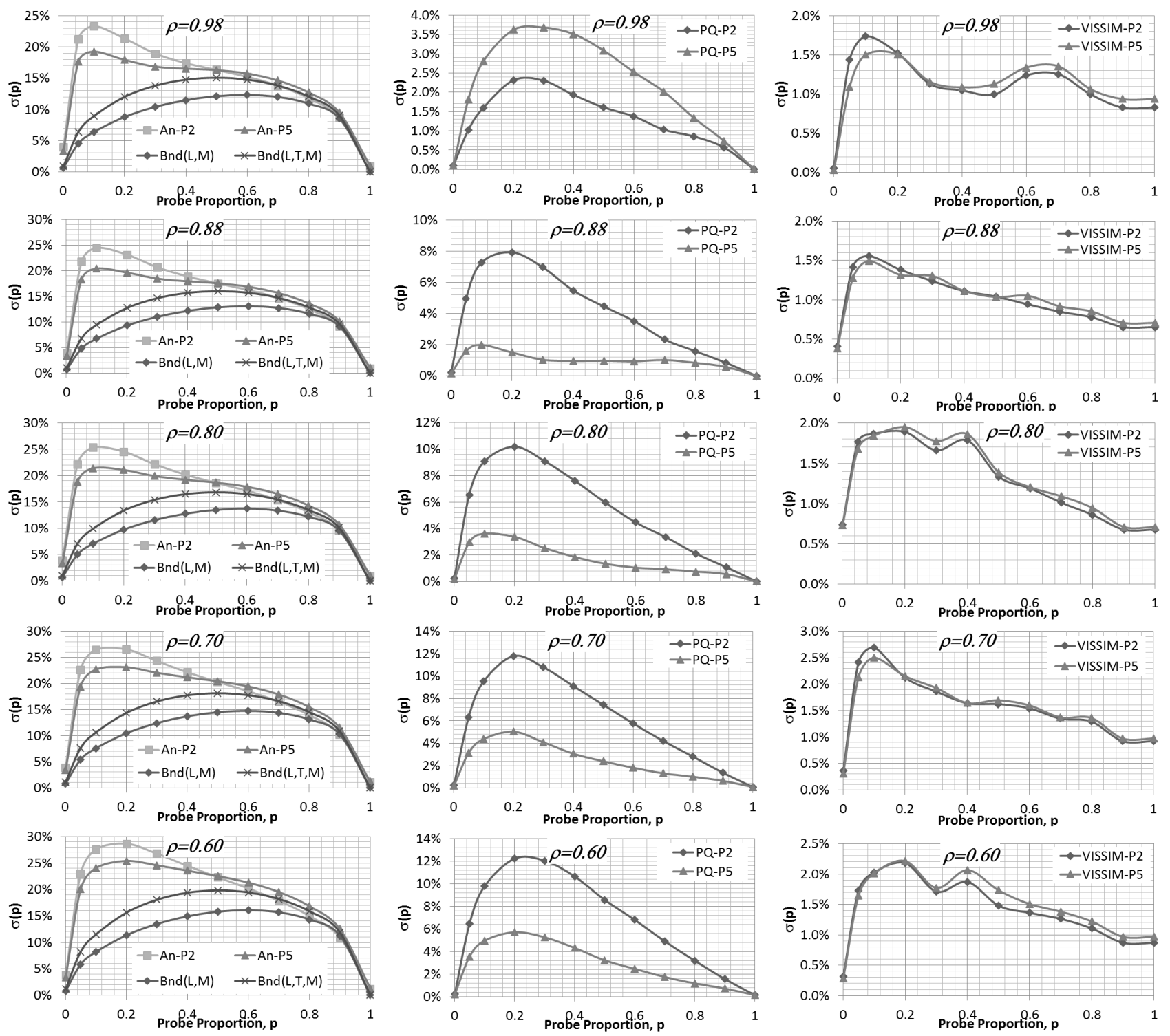

Fig. 7. Steady-state standard deviation of the estimators for $p$ for various $\rho$ levels evaluated by analytical, point queue, and VISSIM simulations

$\hat{p}_{5}$ at very low probe proportions. Due to the differences mentioned earlier, scales of the errors differ by evaluation. Note that figures from analytical formulas are derived for single cycle data to compare the alternatives. For simulations such analysis are rather tedious without omitting the cases when no probe is present in the queue since estimators contain denominators. Therefore, simulation results are used for multiple-cycle comparisons where point queue and VISSIM simulations with $10^{6}$ and $10^{3}$ runs respectively. They are divided into two groups. In the first group, number of cycles are not used as parameters. Instead, $p$ and $\lambda$ are assumed to be constant. In the second group, effect of number of cycles are also shown in Figs. 4 and 5. The overall objective is to find good estimator alternatives for estimating 
the QLs under different scenarios (e.g., arrival rate, probe proportion, and type of applications/number of cycles). From analytical results, $\hat{\lambda}_{2}$ gives the lowest errors. However, it is a biased estimator which can be seen in average QLE results (see Fig. 9). Comparable error values with $\hat{\lambda}_{2}$ can be achieved using $\hat{\lambda}_{4}$ around $p \approx 5 \%$.

\section{Queue Length Estimation with Unknown Parameters}

In this section, queue length estimation with unknown parameters are presented. Estimation errors are shown that are obtained from approximations and simulations.

\subsection{Case $Q=0$}

Estimator of the total queue length at the end of red duration given location, queue joining time of the last PV, and number of PVs in the queue is written as sum of two random variables, $N_{1}$ and $N_{2}$. Random variable $N_{1}$ denotes the queue is up to the last PV and $N_{2}$ is the queue after the last probe vehicle (Eq. (7)). When time index $i$ is the cycle number then the estimator is cycle-to-cycle queue estimator. For an alternative time interval, scanned $(L, T, M)$ can be used for estimation. Certainly, this is a lower bound as some probes may already be left the intersection. As incorporating the counted discharged vehicles, the problem can be alleviated (Hao et al. (2014)). As for implementation, where QL estimated at the end of red can be used for timing the green duration for the signal and lower bound to average queue length for broader signal performance measures. Under the Poisson assumption with known parameters, estimator can be expressed in Eq. (8). Its error can be found using Eq. (9) and be simplified to Eq. (10). Notice that for a multilane formulation, following estimators can be used for signal timing as maximum queue lengths for each lane.

$$
\begin{gathered}
E\left(N \mid L=l, T=t, M=m, Q_{i}=0\right)=E\left(N_{1} \mid L=l, T=t, M=m\right)+E\left(N_{2} \mid L=l, T=t, M=m\right) \\
E\left(N \mid L=l, T=t, M=m, Q_{i}=0\right)=l+(1-p) \lambda(R-t) \\
V\left(D \mid Q_{i}=0\right)=E[V(N \mid L=l, T=t, M=m)] \\
V\left(D \mid Q_{i}=0\right)=\frac{(1-p)\left(1-e^{-\lambda p R}\right)}{p}
\end{gathered}
$$

Without overflow queue, the total queue length with unknown arrival rate and probe proportion can be estimated using Eq. (11). Pairs of the estimators that are considered in numerical examples are listed in Table 4.

$$
E\left(N \mid l, t, m, Q_{i}=0\right)=l+(1-\hat{p}) \hat{\lambda}(R-t)
$$

Performance of these QL estimators can be compared using exact evaluations of their errors by substituting estimators in $E\left(\left[E\left(N \mid l, t, m, Q_{i}=0\right)-N\right]^{2}\right)$. Fig. 8 illustrates the variances derived analytically from Eqs. (D.1)-(D.8). Results closely follow that of overflow queue for $\rho<0.80$. The figure shows the steady-state behavior of QLE errors. When impact of $Q$ is omitted, all of the estimators show similar 
behavior especially for $\rho<0.80$ closely following the true behavior of $V(D)$ s. After $\rho \geq 0.80, V(Q)$ plays a significant role in $V(D)$. Next, subsection 3.2 discusses the performance of the chosen estimators when overflow queue is incorporated into the QLE.

Table 4. Estimators in QLE

\begin{tabular}{lc}
\hline Estimators & $E\left(N \mid l, t, m, Q_{i}=0\right.$ \\
\hline$\hat{p}_{2}, \hat{\lambda}_{2}$ & $l+(l-m)(1-t / R)$ \\
$\hat{p}_{2}, \hat{\lambda}_{3}$ & $l+(1-m / l)[(l) / t](R-t)$ \\
$\hat{p}_{2}, \hat{\lambda}_{4}$ & $l+(1-m / l)[(l-1) / t](R-t)$ \\
$\hat{p}_{2}, \hat{\lambda}_{6}$ & $l+(1-m / l)((l-m) / t+m / R)(R-t)$ \\
\hline$\hat{p}_{5}, \hat{\lambda}_{2}$ & $l+\left(1-\frac{m t}{m t+(l-m) R}\right)(l / R)(R-t)$ \\
$\hat{p}_{5}, \hat{\lambda}_{3}$ & $l+\left(1-\frac{m t}{m t+(l-m) R}\right)(l / t)(R-t)$ \\
$\hat{p}_{5}, \hat{\lambda}_{4}$ & $l+\left(1-\frac{m t}{m t+(l-m) R}\right)((l-1) / t)(R-t)$ \\
$\hat{p}_{5}, \hat{\lambda}_{6}$ & $l+\left(1-\frac{m t}{m t+(l-m) R}\right)((l-m) / t+m / R)(R-t)$ \\
\hline
\end{tabular}
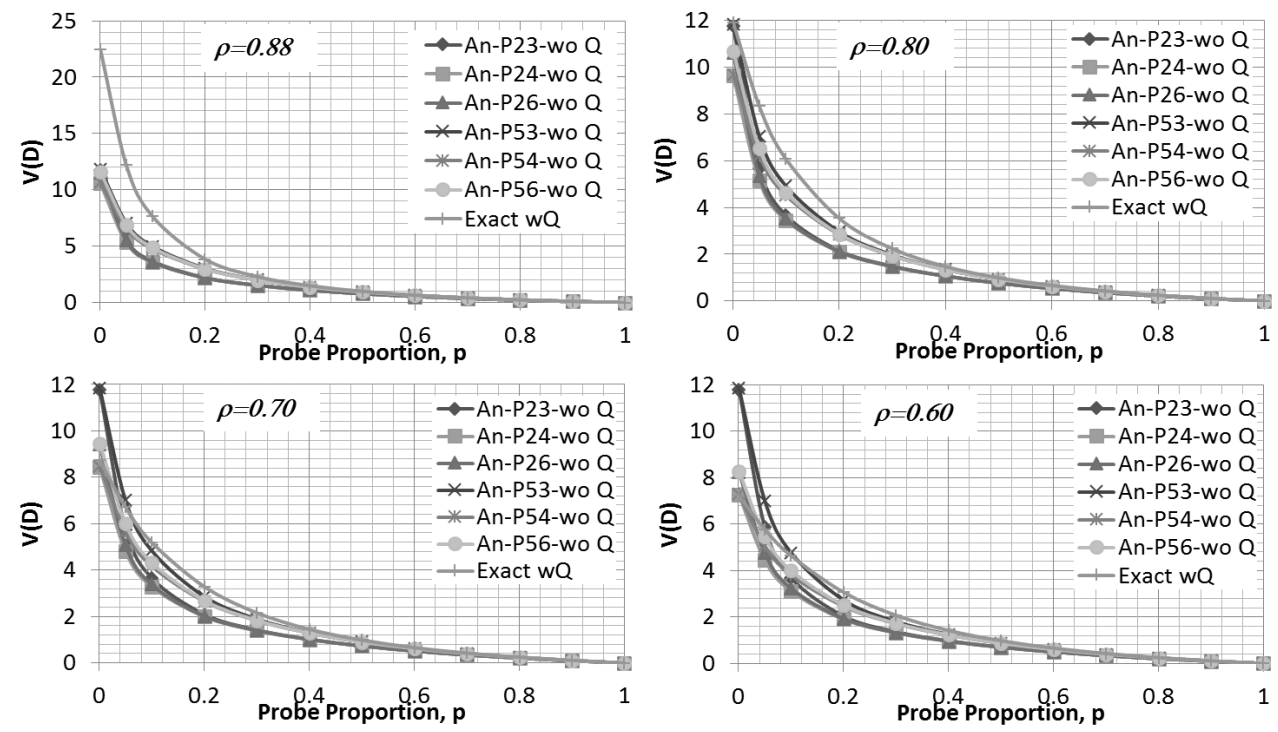

Fig. 8. Steady-state variance of the queue length estimators with unknown parameters without overflow queue for $\rho \leq 0.88$

\subsection{Case $Q \geq 0$}

In this subsection, real-time estimation of cycle-to-cycle as well as steady-state behavior of queue lengths when $Q>0$ are presented. The total $\mathrm{QL}$ at the end of the red duration for this case is written as sum of queues for three different scenarios as shown in Eq. (12). In the equation, $I(l \in Q)$ is indicator function for the case of last $\mathrm{PV}$ is in overflow queue, $I(l \in A)$ represents the last $\mathrm{PV}$ in new arrivals, and $I(l=0)$ denotes when no PV is present in the queue.

$$
E\left(N_{i} \mid L=l, T=t, Q_{i} \geq 0\right)=\left\{\begin{array}{l}
I(l \in Q)\left[l+\hat{\theta}\left(C-t^{\prime}\right)+\hat{\theta} R\right]+ \\
I(l \in A)[l+\hat{\theta} \delta]+ \\
I(l=0)\left[(1-\hat{p})\left(E\left(Q_{i}\right)+\hat{\theta} R\right)\right]
\end{array}\right.
$$


Overflow queue $E\left(Q_{i}\right)=\frac{X i(\hat{\rho}-1)}{4} \sqrt{(\hat{\rho}-1)^{2}+\frac{12\left(\hat{\rho}-\rho_{o}\right)}{X i}}$ is adopted from Akçelik (1980) where $\rho_{o}=0.67+$ $X / 600, X=24$ vehicles per cycle, $\hat{\rho}=\hat{\lambda} C / X$, and $i=1,2,3 \ldots$ denotes the cycle index in this paper for all numerical examples. For $E\left(Q_{i}\right)=E(Q)\left(1-e^{-\beta i}\right)$ from Viti (2006) can also be used which gives very close results (Comert $(2013 \mathrm{~b}))$ where $E(Q)=\frac{3\left(\hat{\rho}-\rho_{o}\right)}{2(1-\hat{\rho})}$. Cycle-to-cycle error of the estimator in Eq. (12) i.e., $V\left(D_{i}\right)$ can be given as in Eq. (13) with $V\left(Q_{i}\right)=\left[E(Q)(\hat{\rho}+(1-\hat{p}) / 0.15)+\left(\sqrt{\hat{\rho} X i}-\sigma_{Q_{e}}\right) e^{-\beta i}\right]^{2}$ from Viti (2006). Where $\sigma_{Q_{e}}$ can be calculated from $\sigma_{Q_{e}}=E(Q)(\hat{\rho}+(1-\hat{\rho}) / 0.15)$. Steady-state version is also written simply in Eq. (17).

$$
V\left(D_{i} \mid Q_{i} \geq 0\right)=\left\{\begin{array}{l}
P(l \in Q)\left[\hat{\theta}\left(C-E\left(T^{\prime}\right)\right)+\hat{\theta} R\right]+ \\
P(l \in A)\left[(1-\hat{p})\left(1-e^{-p \hat{\lambda} R}\right) / \hat{p}\right]+ \\
P(l=0)\left[(1-\hat{p})\left(V\left(Q_{i}\right)+\hat{\theta} R\right)\right]
\end{array}\right.
$$

These scenarios in Eq. (14) are calculated in the simulations easily by conditional statements. In analytical formulas, scenario probabilities are approximated by Eq. (15). After testing various weights, $E(Q)$ (or $\left.E\left(Q_{i}\right)\right)$ in Eq. (15) is weighted by a quadratic function obtained by fitting actual versus approximate estimation error values to reduce $P(L=0)$ slowly at high $\rho$ values when probe proportion increases.

$$
P(L=l) \approx\left\{\begin{array}{l}
{\left[1-\sum_{q=0}(1-\hat{p})^{q} P(Q=q)\right] e^{-\hat{\lambda} R \hat{p}}, l \in Q} \\
1-\sum_{a=0}(1-\hat{p})^{a} P(A=a), l \in A \\
\sum_{q=0}(1-\hat{p})^{q} P(Q=q) e^{-\hat{\lambda} R \hat{p}}, l=0
\end{array}\right.
$$

Where, scenario probabilities are approximated by,

$$
P(L=l)=\left\{\begin{array}{l}
{\left[1-e^{-p\left[\left(9.87 p^{2}-4.62 p+0.991\right) E(Q)\right]}\right] e^{-p \lambda R}, l \in Q} \\
e^{-p \lambda R}, l \in A \\
e^{-p\left[\lambda R\left(9.87 p^{2}-4.62 p+0.991\right) E(Q)\right]}, l=0
\end{array}\right.
$$

Fig. 9 shows the steady-state behavior of expected values of QLEs from Eqs. (D.1)-(D.8) in Table 4. From the figure, effect of biasness in the estimators can be seen. As discussed earlier, $\hat{\lambda}_{2}$ has low error for single cycle applications, but, it does not give true average total queue length especially at low probe proportion levels. Estimators $\hat{\lambda}_{4}, \hat{\lambda}_{6}, \hat{p}_{2}$, and $\hat{p}_{5}$ seem to be more reasonable choices.

$$
E(N)=\left\{\begin{array}{l}
P(l \in Q)\left[E(L)+\hat{\theta}\left(C-E\left(T^{\prime}\right)\right)+\hat{\theta} R\right]+ \\
P(l \in A)[\hat{\lambda} R]+ \\
I(l=0)[(1-\hat{p})(E(Q)+\hat{\theta} R)]
\end{array} \quad V(D \mid Q \geq 0)=\left\{\begin{array}{l}
P(l \in Q)\left[\hat{\theta}\left(C-E\left(T^{\prime}\right)\right)+\hat{\theta} R\right]+ \\
P(l \in A)\left[(1-\hat{p})\left(1-e^{-p \hat{\lambda} R}\right) / \hat{p}\right]+ \\
P(l=0)[(1-\hat{p})(V(Q)+\theta R)]
\end{array}\right.\right.
$$

In Fig. 9, steady-state values of the expected value of queue length estimators are given when no low level parameter is assumed to be known. Especially for high $\rho \geq 0.88$ levels, at about $p=5 \%$, true average 

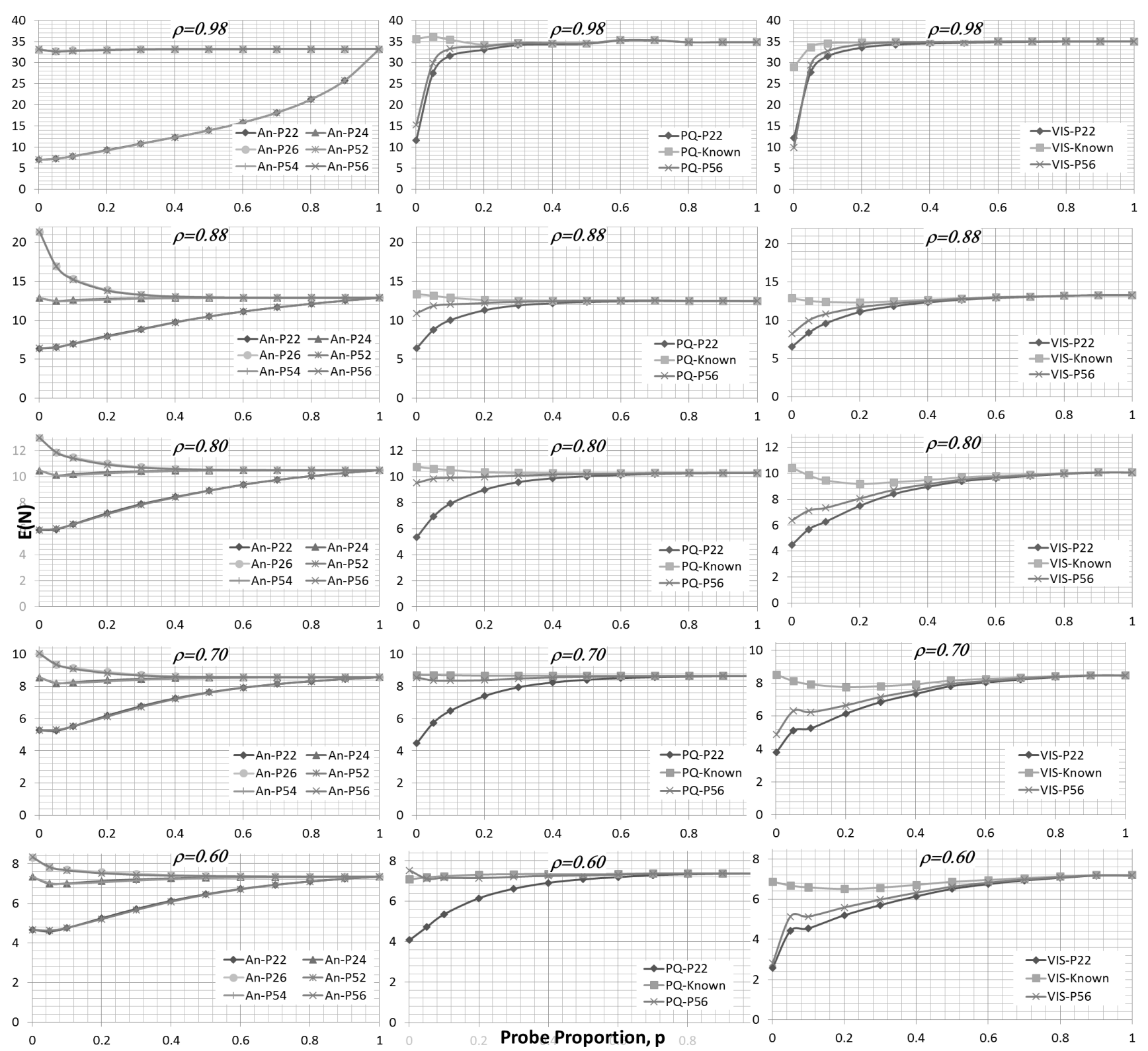

Fig. 9. Steady-state expected value of the queue length estimators with unknown parameters

queue length can be achieved by the estimators. For $\rho<0.88$, since the average queue size is small, the difference stays within \pm 2 vehicles even with worse performance by $\left(\hat{p}_{2}, \hat{\lambda}_{2}\right)$. Other estimators are close. Specifically, combination of $\left(\hat{p}_{5}, \hat{\lambda}_{6}\right)$ provides very close results for every $\rho$ and $p$ levels.

Table 5 summarizes percent differences of $E\left(N_{i}\right)$ and $C V_{i}=\sqrt{V\left(D_{i}\right)} / E\left(N_{i}\right)$ that are cycle-to-cycle behavior (up to 50 cycles) calculated with $\left(\hat{p}_{2}, \hat{\lambda}_{2}\right)$ and $\left(\hat{p}_{5}, \hat{\lambda}_{6}\right)$ from true average queue length values for $\rho=0.98,0.88,0.70$ and $p=0.001,0.05,0.10,0.20,0.30,0.50$ values. Fig. 10 as an example demonstrates low $p$ and high $\rho$ values. Objective of showing these two combinations is representation of the least information $L=l$ and the most probe information demanded $(L, M, T)$ by parameter estimators. When $p=0.001$, the estimators are not able to point the true average queue lengths before high number of cycles 

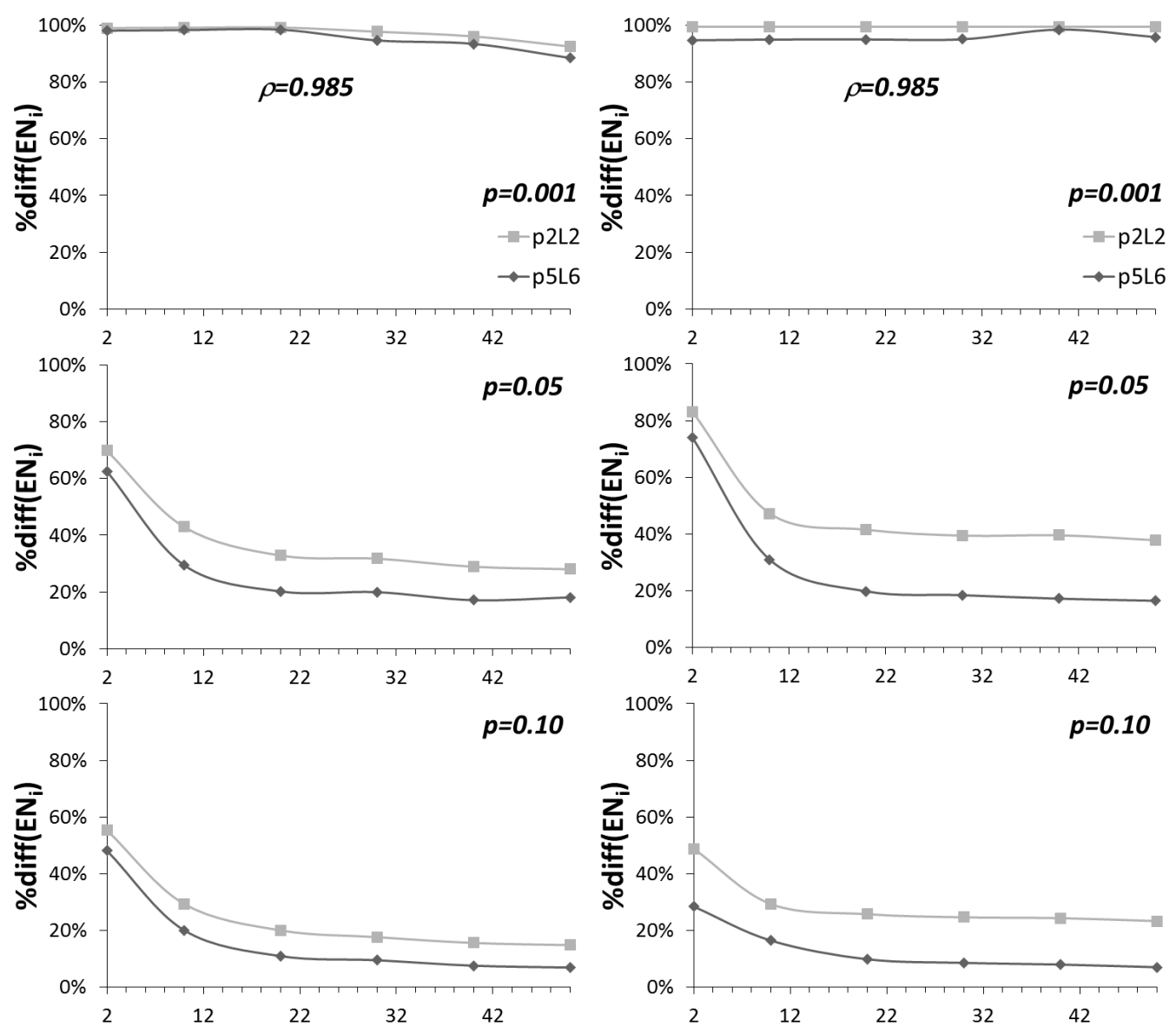

Fig. 10. Percent difference between cycle-to-cycle expected value of the queue length estimators with unknown parameters and true queue lengths

which can be supported by Fig. 9 for $\left(\hat{p}_{5}, \hat{\lambda}_{6}\right)$. When $p<0.50$, within $5 \%$ of the true queue length values are only obtained after $p=0.20$ or after 50 cycles. Likewise, $30 \%$ penetration rate was recommended by Ban et al. (2011); Goodall et al. (2014) and according to Hao et al. (2014), penetration rates $\geq 10 \%$ is able to provide mean absolute error within \pm 3 vehicles in queue length estimation.

In Fig. 11, steady-state values of the estimation errors are given when no low level parameter is assumed to be known. Simulation results show queue length estimations and errors are calculated for different true values. Analytical results can be used to evaluate steady-state behavior of such estimators for different parameter values under assumed conditions and distributions without any simulations. Overall, estimators with unknown parameters are closely following the true $V(D)$ values with $\left(\hat{p}_{2}, \hat{\lambda}_{2}\right),\left(\hat{p}_{2}, \hat{\lambda}_{4}\right)$, $\left(\hat{p}_{2}, \hat{\lambda}_{6}\right),\left(\hat{p}_{5}, \hat{\lambda}_{4}\right)$, and $\left(\hat{p}_{5}, \hat{\lambda}_{6}\right)$. Again for cycle-to-cycle performance evaluations, $\left(\hat{p}_{2}, \hat{\lambda}_{2}\right)$ and $\left(\hat{p}_{5}, \hat{\lambda}_{6}\right)$, are shown.

Derived from Table 5, Fig. 12 illustrates percent differences of relative standardized errors, $C V_{i}$ with unknown parameters up to 50 cycles. From the figures, at all $p$ and $\rho$ levels, queue estimators perform well staying within $\pm 5 \%$. For high $\rho$ and low $p$ values of the true values $\left(\hat{p}_{5}, \hat{\lambda}_{6}\right)$ can be used. From 
Table 5. Up to $i=50$ cycle-to-cycle $\% C V_{i}$ and $\% E N_{i}$ differences of the queue length estimators with unknown parameters and true queue lengths

\begin{tabular}{|c|c|c|c|c|c|c|c|c|c|c|c|c|c|}
\hline & \multicolumn{4}{|c|}{$\% \Delta C V_{i} \hat{p}_{2} \hat{\lambda}_{2}$} & \multicolumn{3}{|c|}{$\% \Delta C V_{i} \hat{p}_{5} \hat{\lambda}_{6}$} & \multicolumn{3}{|c|}{$\% \Delta E N_{i} \hat{p}_{2} \hat{\lambda}_{2}$} & \multicolumn{3}{|c|}{$\% \Delta E N_{i} \hat{p}_{5} \hat{\lambda}_{6}$} \\
\hline & Cycles & $\rho=0.985$ & $\rho=0.88$ & $\rho=0.70$ & $\rho=0.985$ & $\rho=0.88$ & $\rho=0.70$ & $\rho=0.985$ & $\rho=0.88$ & $\rho=0.70$ & $\rho=0.985$ & $\rho=0.88$ & $\rho=0.70$ \\
\hline \multirow{6}{*}{$\mathrm{p}=0.1 \%$} & 2 & $1 \%$ & $-1 \%$ & $0 \%$ & $1 \%$ & $-1 \%$ & $0 \%$ & $99 \%$ & $100 \%$ & $98 \%$ & $98 \%$ & $95 \%$ & $98 \%$ \\
\hline & 10 & $-2 \%$ & $-2 \%$ & $0 \%$ & $-2 \%$ & $-3 \%$ & $0 \%$ & $99 \%$ & $100 \%$ & $98 \%$ & $98 \%$ & $95 \%$ & $98 \%$ \\
\hline & 20 & $-3 \%$ & $-1 \%$ & $-1 \%$ & $-3 \%$ & $-2 \%$ & $-2 \%$ & $99 \%$ & $100 \%$ & $98 \%$ & $98 \%$ & $95 \%$ & $98 \%$ \\
\hline & 30 & $-3 \%$ & $-2 \%$ & $-1 \%$ & $-3 \%$ & $-3 \%$ & $-2 \%$ & $98 \%$ & $100 \%$ & $98 \%$ & $95 \%$ & $95 \%$ & $98 \%$ \\
\hline & 40 & $-3 \%$ & $-2 \%$ & $-2 \%$ & $-2 \%$ & $-3 \%$ & $-3 \%$ & $96 \%$ & $100 \%$ & $98 \%$ & $93 \%$ & $99 \%$ & $98 \%$ \\
\hline & 50 & $-2 \%$ & $-1 \%$ & $-2 \%$ & $-2 \%$ & $-3 \%$ & $-4 \%$ & $93 \%$ & $100 \%$ & $98 \%$ & $89 \%$ & $96 \%$ & $98 \%$ \\
\hline \multirow{6}{*}{$\mathrm{p}=5 \%$} & 2 & $-6 \%$ & $-6 \%$ & $-12 \%$ & $-5 \%$ & $-5 \%$ & $-15 \%$ & $70 \%$ & $83 \%$ & $80 \%$ & $63 \%$ & $74 \%$ & $68 \%$ \\
\hline & 10 & $-7 \%$ & $-9 \%$ & $-12 \%$ & $-6 \%$ & $-10 \%$ & $-15 \%$ & $43 \%$ & $47 \%$ & $52 \%$ & $29 \%$ & $31 \%$ & $29 \%$ \\
\hline & 20 & $-7 \%$ & $-8 \%$ & $-10 \%$ & $-5 \%$ & $-8 \%$ & $-13 \%$ & $33 \%$ & $42 \%$ & $43 \%$ & $20 \%$ & $20 \%$ & $16 \%$ \\
\hline & 30 & $-7 \%$ & $-7 \%$ & $-9 \%$ & $-5 \%$ & $-6 \%$ & $-12 \%$ & $32 \%$ & $39 \%$ & $43 \%$ & $20 \%$ & $18 \%$ & $13 \%$ \\
\hline & 40 & $-6 \%$ & $-7 \%$ & $-7 \%$ & $-4 \%$ & $-6 \%$ & $-9 \%$ & $29 \%$ & $40 \%$ & $43 \%$ & $17 \%$ & $17 \%$ & $20 \%$ \\
\hline & 50 & $-6 \%$ & $-6 \%$ & $-7 \%$ & $-3 \%$ & $-6 \%$ & $-8 \%$ & $28 \%$ & $38 \%$ & $43 \%$ & $18 \%$ & $16 \%$ & $16 \%$ \\
\hline \multirow{6}{*}{$\mathrm{p}=10 \%$} & 2 & $7 \%$ & $-9 \%$ & $-8 \%$ & $6 \%$ & $-8 \%$ & $-13 \%$ & $55 \%$ & $49 \%$ & $62 \%$ & $48 \%$ & $28 \%$ & $44 \%$ \\
\hline & 10 & $-8 \%$ & $-8 \%$ & $-9 \%$ & $-6 \%$ & $-6 \%$ & $-10 \%$ & $29 \%$ & $29 \%$ & $33 \%$ & $20 \%$ & $16 \%$ & $18 \%$ \\
\hline & 20 & $-5 \%$ & $-7 \%$ & $-9 \%$ & $-3 \%$ & $-7 \%$ & $-11 \%$ & $20 \%$ & $26 \%$ & $33 \%$ & $11 \%$ & $10 \%$ & $17 \%$ \\
\hline & 30 & $-4 \%$ & $-6 \%$ & $-7 \%$ & $-2 \%$ & $-5 \%$ & $-7 \%$ & $18 \%$ & $25 \%$ & $31 \%$ & $10 \%$ & $9 \%$ & $10 \%$ \\
\hline & 40 & $-3 \%$ & $-6 \%$ & $-7 \%$ & $-1 \%$ & $-4 \%$ & $-7 \%$ & $16 \%$ & $24 \%$ & $30 \%$ & $8 \%$ & $8 \%$ & $8 \%$ \\
\hline & 50 & $-3 \%$ & $-5 \%$ & $-6 \%$ & $-1 \%$ & $-3 \%$ & $-5 \%$ & $15 \%$ & $23 \%$ & $29 \%$ & $7 \%$ & $7 \%$ & $7 \%$ \\
\hline \multirow{6}{*}{$\mathrm{p}=20 \%$} & 2 & $-1 \%$ & $-3 \%$ & $3 \%$ & $0 \%$ & $-1 \%$ & $0 \%$ & $24 \%$ & $8 \%$ & $12 \%$ & $13 \%$ & $0 \%$ & $4 \%$ \\
\hline & 10 & $-3 \%$ & $-5 \%$ & $-7 \%$ & $-2 \%$ & $-5 \%$ & $-7 \%$ & $9 \%$ & $13 \%$ & $16 \%$ & $3 \%$ & $5 \%$ & $5 \%$ \\
\hline & 20 & $-3 \%$ & $-2 \%$ & $-5 \%$ & $-1 \%$ & $-2 \%$ & $-5 \%$ & $9 \%$ & $11 \%$ & $17 \%$ & $3 \%$ & $3 \%$ & $6 \%$ \\
\hline & 30 & $-1 \%$ & $-3 \%$ & $-6 \%$ & $-1 \%$ & $-2 \%$ & $-5 \%$ & $6 \%$ & $12 \%$ & $17 \%$ & $2 \%$ & $4 \%$ & $4 \%$ \\
\hline & 40 & $-1 \%$ & $-2 \%$ & $-4 \%$ & $0 \%$ & $-1 \%$ & $-4 \%$ & $6 \%$ & $11 \%$ & $15 \%$ & $2 \%$ & $3 \%$ & $3 \%$ \\
\hline & 50 & $0 \%$ & $-2 \%$ & $-4 \%$ & $0 \%$ & $-1 \%$ & $-3 \%$ & $6 \%$ & $11 \%$ & $15 \%$ & $2 \%$ & $4 \%$ & $3 \%$ \\
\hline \multirow{6}{*}{$\mathrm{p}=30 \%$} & 2 & $1 \%$ & $-7 \%$ & $-7 \%$ & $0 \%$ & $-6 \%$ & $-8 \%$ & $6 \%$ & $6 \%$ & $-1 \%$ & $-2 \%$ & $3 \%$ & $-5 \%$ \\
\hline & 10 & $-1 \%$ & $-2 \%$ & $-2 \%$ & $-1 \%$ & $-6 \%$ & $-3 \%$ & $4 \%$ & $7 \%$ & $8 \%$ & $2 \%$ & $2 \%$ & $2 \%$ \\
\hline & 20 & $-1 \%$ & $-2 \%$ & $-3 \%$ & $0 \%$ & $-1 \%$ & $-3 \%$ & $4 \%$ & $6 \%$ & $9 \%$ & $1 \%$ & $2 \%$ & $3 \%$ \\
\hline & 30 & $-1 \%$ & $-2 \%$ & $-3 \%$ & $0 \%$ & $-2 \%$ & $-2 \%$ & $3 \%$ & $6 \%$ & $9 \%$ & $1 \%$ & $2 \%$ & $2 \%$ \\
\hline & 40 & $-1 \%$ & $-1 \%$ & $-3 \%$ & $0 \%$ & $-1 \%$ & $-2 \%$ & $3 \%$ & $6 \%$ & $9 \%$ & $1 \%$ & $1 \%$ & $1 \%$ \\
\hline & 50 & $-1 \%$ & $-1 \%$ & $-3 \%$ & $0 \%$ & $-1 \%$ & $-4 \%$ & $3 \%$ & $6 \%$ & $8 \%$ & $1 \%$ & $1 \%$ & $2 \%$ \\
\hline \multirow{6}{*}{$\mathrm{p}=50 \%$} & 2 & $0 \%$ & $0 \%$ & $-1 \%$ & $-1 \%$ & $0 \%$ & $-1 \%$ & $1 \%$ & $2 \%$ & $2 \%$ & $0 \%$ & $0 \%$ & $0 \%$ \\
\hline & 10 & $-1 \%$ & $-1 \%$ & $-1 \%$ & $0 \%$ & $-1 \%$ & $-1 \%$ & $2 \%$ & $2 \%$ & $3 \%$ & $1 \%$ & $0 \%$ & $1 \%$ \\
\hline & 20 & $0 \%$ & $-1 \%$ & $-1 \%$ & $0 \%$ & $0 \%$ & $-1 \%$ & $1 \%$ & $2 \%$ & $3 \%$ & $0 \%$ & $1 \%$ & $1 \%$ \\
\hline & 30 & $0 \%$ & $-1 \%$ & $-1 \%$ & $0 \%$ & $0 \%$ & $-1 \%$ & $1 \%$ & $2 \%$ & $3 \%$ & $0 \%$ & $1 \%$ & $1 \%$ \\
\hline & 40 & $0 \%$ & $0 \%$ & $-1 \%$ & $0 \%$ & $0 \%$ & $-1 \%$ & $1 \%$ & $2 \%$ & $3 \%$ & $0 \%$ & $1 \%$ & $1 \%$ \\
\hline & 50 & $0 \%$ & $0 \%$ & $-1 \%$ & $0 \%$ & $-1 \%$ & $-1 \%$ & $1 \%$ & $2 \%$ & $3 \%$ & $0 \%$ & $1 \%$ & $1 \%$ \\
\hline
\end{tabular}

the table, for low $\rho$ and low $p$ values of the true values $\left(\hat{p}_{2}, \hat{\lambda}_{2}\right)$ can be utilized. With less than 10 cycles of information, the differences from the true $\% \mathrm{CV}$ levels stay within $\pm 10 \%$. For all three $\rho$ values for $p=0.001$ the relative error stays $\pm 5 \%$ which is desirable. For $p=0.05$, the errors get close to $\pm \% 5$ level at about 50 cycles. After $p=0.10$, accuracy is acceptable especially for $\rho=0.98$ and $\rho=0.88$. Fig. 13 gives linear fits that regress the steady-state parameter estimations (i.e., $\hat{p}_{5}$ and $\hat{\lambda}_{6}$ ) and queue length estimation errors of analytical approximations, point queue, and VISSIM evaluations versus exact values from VISSIM. The estimation errors look pretty consistent with high $R^{2}=0.94$ values when overflow queue is included. When $Q$ is omitted and $\rho \leq 0.88$, simple analytical model can also be used as it is able to give close results $R^{2}=0.86$. For parameter estimators, $p$ and $\lambda$ evaluations agree with the true values with lowest $R^{2}=0.97$ and $R^{2}=0.74$ respectively. It can be seen from the figure that in VISSIM evaluations especially at low $p$ and $\rho$ values show some dispersed values. 

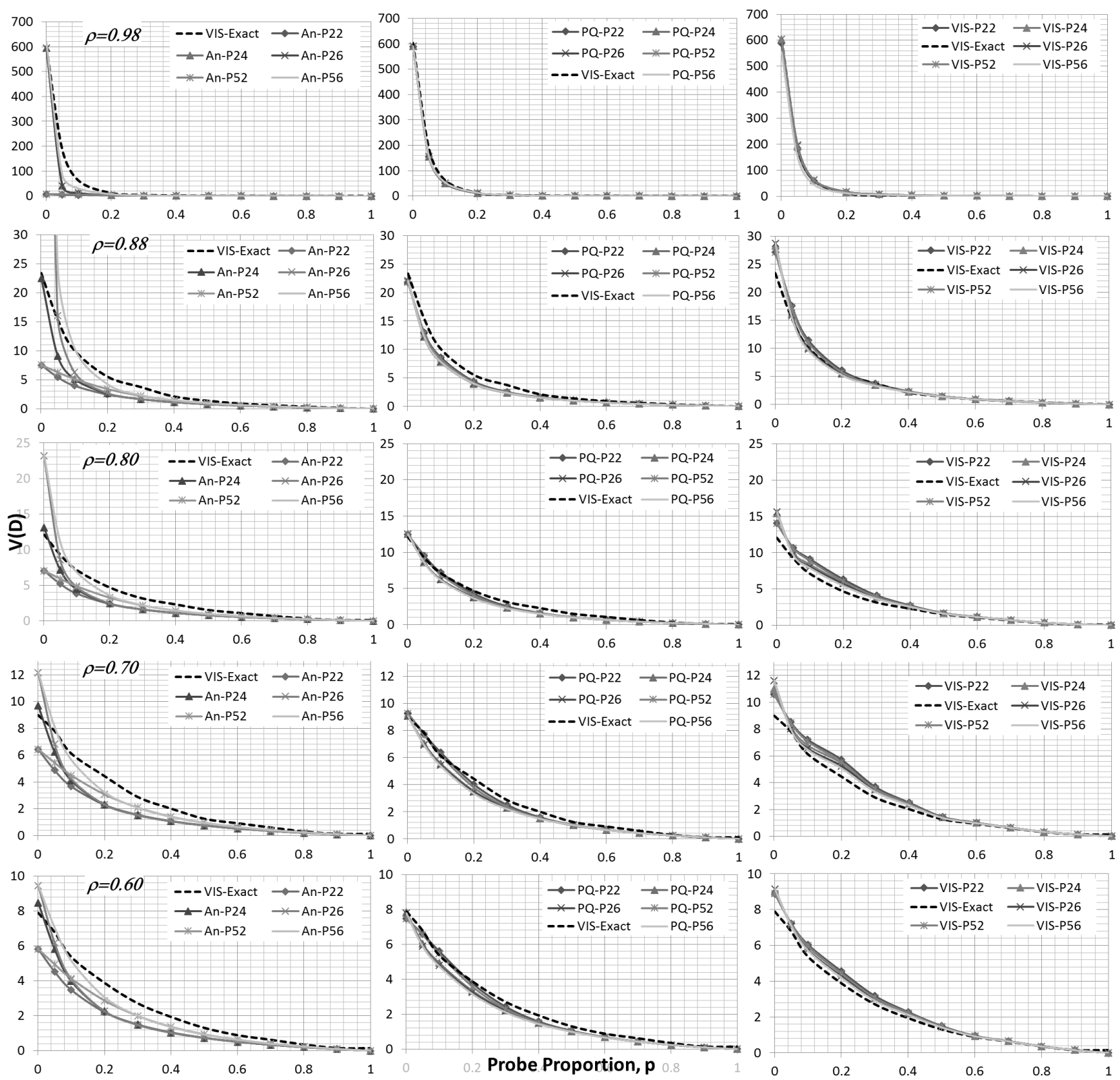

Fig. 11. Steady-state $V(D)$ of the queue length estimators for with unknown parameters for different $\rho$ and $p$ values

\section{Conclusions}

In this paper, stochastic models for queue length estimation with unknown primary parameters from PVs at traffic intersections are derived. Developed models use only primary signal parameters and probe information. Several alternatives for arrival rate $\lambda$ and probe proportion $p$ are given as $\hat{\lambda}_{1}=m / p R$, $\hat{\lambda}_{2}=l / R, \hat{\lambda}_{3}=l / t, \hat{\lambda}_{4}=(l-1) / t, \hat{\lambda}_{5}=l /(t+p(R-t)), \hat{\lambda}_{6}=(l-m) / t+m / R$ and $\hat{p}_{1}=m /(\lambda R)$, $\hat{p}_{2}=m / l, \hat{p}_{3}=1 /(\lambda(R-t)), \hat{p}_{4}=t /[(R-t)(l-1)], \hat{p}_{5}=m t /(m t+(l-m) R)$. Numerical results show that $\hat{\lambda}_{4}$ and $\hat{\lambda}_{6}$ provide the lowest errors for all true $\lambda$ tested for $p \leq 5 \%$ levels with 10 cycles of data. For probe proportion, estimators $\hat{p}_{2}$ and $\hat{p}_{5}$ are found to be better alternatives. Instead, simple biased ones 

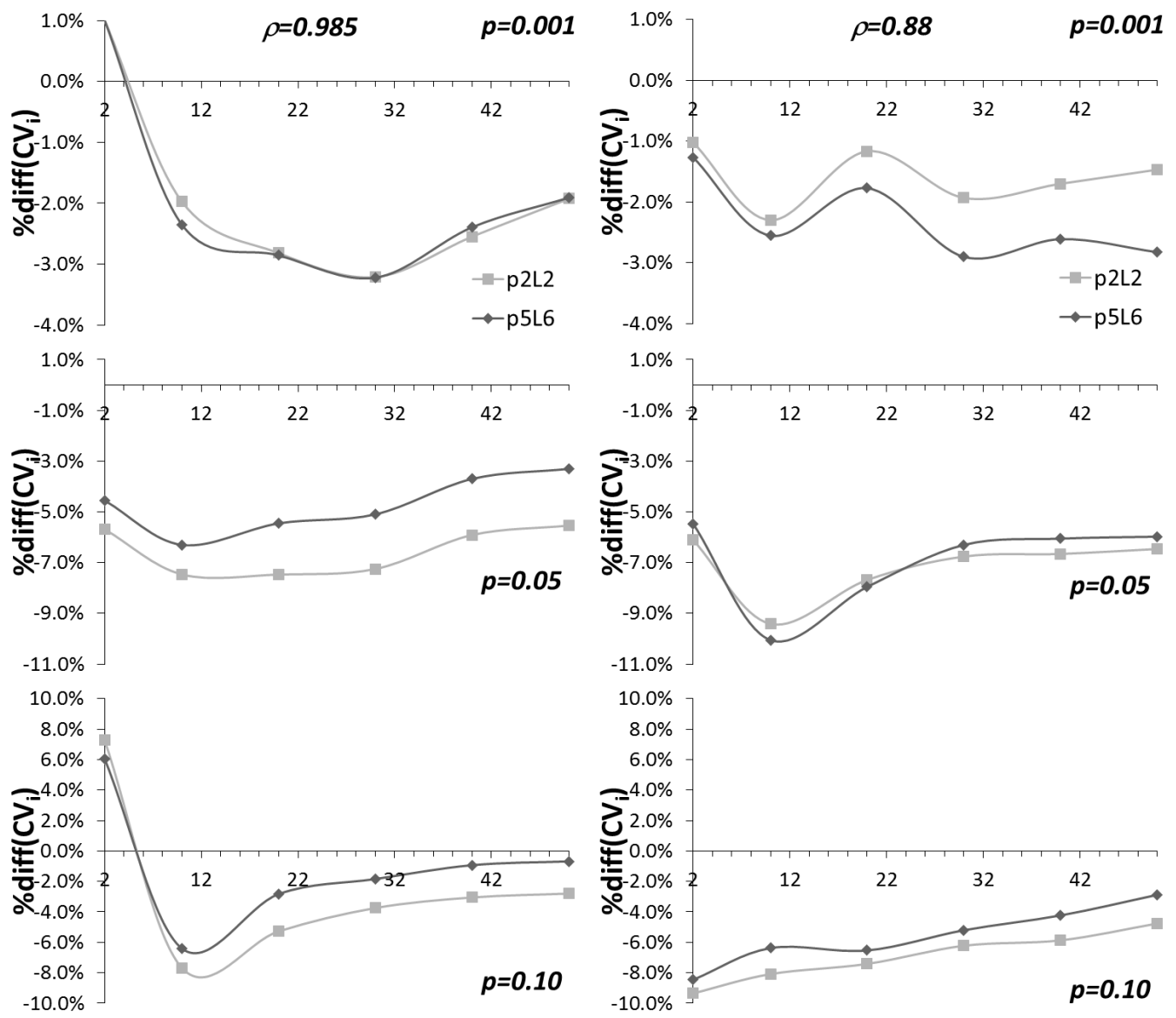

Fig. 12. Up to $i=50$ cycle-to-cycle $\% C V_{i}$ differences with unknown parameters at $\rho=0.98,0.88$ and $p=0.001,0.05,0.10$
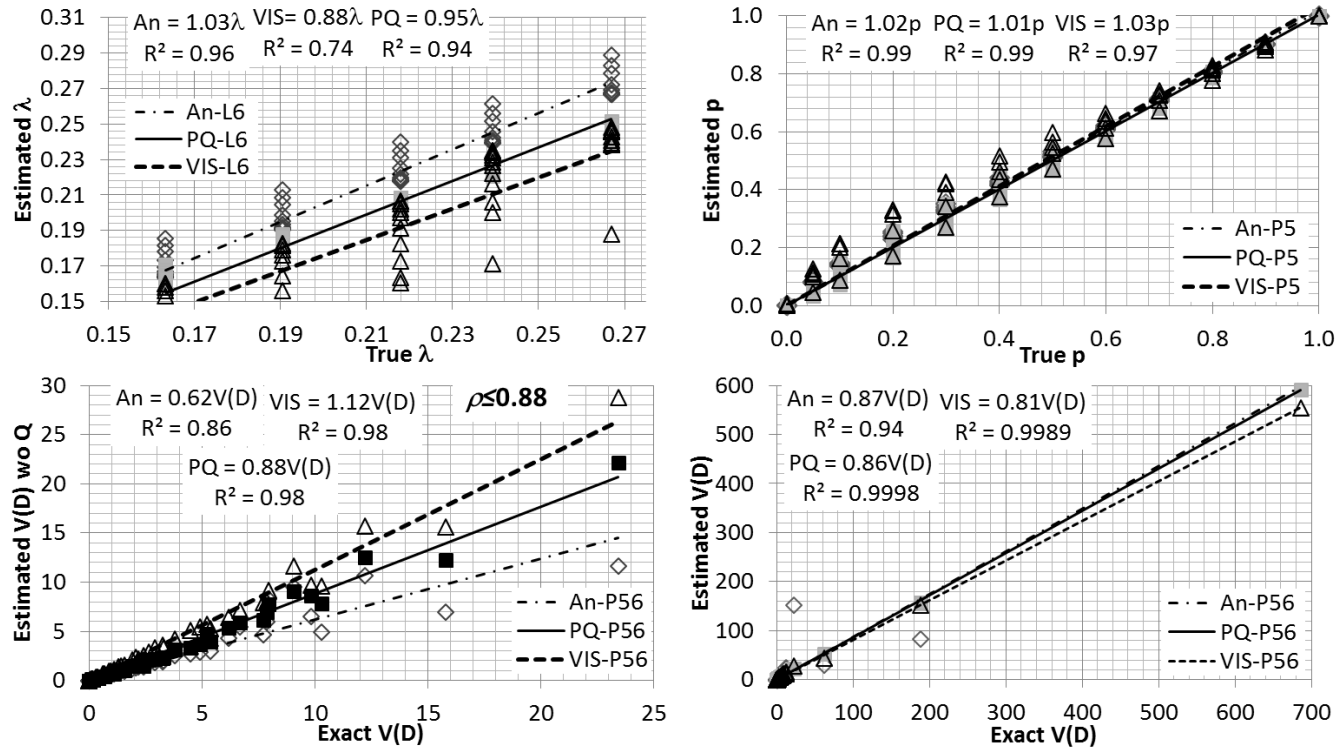

Fig. 13. Steady-state parameter and queue length estimation errors versus true values

$\hat{p}_{2}$ and $\hat{\lambda}_{2}$ can be used for any true $p$ and $\lambda$ values after $p>5 \%$. For low $p$ values such as $0.1 \%$, large number of cycles of data is required by $\hat{\lambda}$ s which are essential to estimate overflow queue and volume-to- 
capacity ratios. Estimators for $p$ do not demand these conditions. When there is no probe in the queue simply $\hat{p}=0$. They are able to point true $p$ at low penetration levels. Performance of the estimators for multiple cycles of data is also discussed whenever no probe information is available. Cycle-to-cycle queue estimators are able to capture the exact errors within $\pm 5 \%$ of coefficient of variations with less than 5 cycles of probe data at $p=0.1 \%$ for all $\lambda$ levels. The differences further reduces as $p$ increases. Especially for high $\rho$ values, estimators follow true values closely as likelihood of a probe vehicle being in the queue is greater. Some of the future work can be listed as evaluating other possible estimators, and different arrival distributions.

\section{Acknowledgments}

This paper is based upon work supported by the National Science Foundation (NSF) under Grant No. 1238705 and partially supported by NSF Grant No. 1436222. Any opinions, findings, and conclusions or recommendations expressed in this paper are those of the author and do not necessarily reflect the views of the NSF. The author would like to thank to Editor Prof. Jose Oliveira and anonymous reviewers for their insightful comments which greatly improved the paper. Author also thanks Prof. Negash Begashaw for his valuable feedback on presentation of the models.

\section{Appendix A. Derivation of Parameter Estimators}

In this section, step-by-step derivations of the parameter estimators are given. First, joint distributions of probe vehicles are derived for finding the maximum likelihood estimators. Marginal distributions are from Eqs. (1)-(3).

$$
\begin{array}{r}
f(t \mid l)=\frac{(\lambda)(\lambda t)^{l-1} e^{-t \theta} e^{-p \lambda(R-t)}}{(l-1) ! \int_{0}^{R} \frac{(\lambda)(\lambda t)^{l-1} e^{-t \theta} e^{-p \lambda(R-t)} d t}{(l-1) !}} \\
f(t, l)=\left[\frac{(\lambda)(\lambda t)^{l-1} e^{-t \theta} e^{-p \lambda(R-t)}}{(l-1) ! \int_{0}^{R} \frac{(\lambda)(\lambda t)^{l-1} e^{-t \theta} e^{-p \lambda(R-t)}}{(l-1)} d t}\right]\left[\frac{p}{(1-p)^{l}} \sum_{n=l}^{\infty} \frac{e^{-\lambda R}(\theta R)^{n}}{n !}\right] \\
f(t, l)=\left[\frac{(\theta)(\theta t)^{l-1} e^{-t \theta}}{(l-1) ! \sum_{n=l}^{\infty} \frac{(\theta R)^{n} e^{-\theta R}}{n !}}\right]\left[\frac{p}{(1-p)^{l}} \sum_{n=l}^{\infty} \frac{e^{-\lambda R}(\theta R)^{n}}{n !}\right]=\frac{(\lambda p)(\lambda t)^{l-1} e^{-t \theta} e^{-p \lambda R}}{(l-1) !}
\end{array}
$$

The joint probability density function of $f(t, l, m)$ is obtained by $f(t, l, m)=f(t \mid l, m) P(L=l, M=m)$. After simplifications $f(t, l, m)$ can be written as,

$$
\begin{array}{r}
P(L=l, M=m)=\sum_{n=l}^{\infty} \frac{\left(\begin{array}{c}
l-1 \\
m-1
\end{array}\right) p^{m}(1-p)^{n-m} e^{-\lambda R}(\lambda R)^{n}}{n !} \\
f(t \mid l, m)=\frac{(\theta)(\theta t)^{l-1} e^{-t \theta}}{(l-1) ! \sum_{k=l}^{\infty} \frac{(\theta R)^{k} e^{-\theta R}}{k !}}
\end{array}
$$




$$
f(t, l, m)=\left\{\begin{array}{l}
\frac{p^{m}(1-p)^{-m} \theta(\theta t)^{l-1} e^{-t \theta} e^{-p \lambda R}}{(m-1) !(l-m) !}, M>0 \\
e^{-p \lambda R}, M=0
\end{array}\right.
$$

Using only $M$, estimators $\hat{p}_{1}$ and $\hat{\lambda}_{1}$ are given as:

$$
\begin{array}{r}
f(m)=\frac{(\lambda p R)^{m} e^{-\lambda p R}}{(m) !} \\
\log (f(m))=m \log (\lambda p R)-\lambda p R-\log (m !) \\
\frac{\partial \log (f(m))}{\partial p}=m / p-\lambda R=0 \\
\hat{p}_{1}=m /(\lambda R) \\
\frac{\partial \log (f(m))}{\partial \lambda}=m / \lambda-p R=0 \\
\hat{\lambda}_{1}=m /(p R)
\end{array}
$$

Using $L, T$, estimators $\hat{p}_{3}, \hat{p}_{4}, \hat{\lambda}_{3}, \hat{\lambda}_{4}$, and $\hat{\lambda}_{5}$ are given as:

$$
\begin{array}{r}
f(t, l)=\frac{(\lambda p)(\lambda t)^{l-1} e^{-t \theta} e^{-p \lambda R}}{(l-1) !} \\
\log (f(t, l))=\log (\lambda p)+(l-1) \log (\lambda t)-\lambda p R-t \theta-\log ((l-1) !) \\
\frac{\partial \log (f(t, l))}{\partial p}=1 / p-\lambda R+t \lambda=0 \\
\hat{p}_{3}=1 /(\lambda(R-t)) \\
\hat{p}_{4}=t /((l-1)(R-t)) \\
\frac{\partial \log (f(t, l))}{\partial \lambda}=1 / \lambda+(l-1) / \lambda-t+t p-p R=0 \\
\hat{\lambda}_{5}=l /(t+p(R-t)) \\
\hat{\lambda}_{4}=(l-1) / t \\
\hat{\lambda}_{3}=l / t
\end{array}
$$

Using $L, T, M$, estimators $\hat{p}_{5}$ and $\hat{\lambda}_{6}$ are given as:

$$
\begin{array}{r}
f(t, l, m)=\frac{p^{m}(1-p)^{-m} \theta(\theta t)^{l-1} e^{-t \theta} e^{-p \lambda R}}{(m-1) !(l-m) !} \\
\log (f(t, l, m))=m \log (p)-m \log (1-p)+\log (\theta)+(l-1) \log (\theta t)-\lambda p R-t \theta \\
-\log ((l-m) !)-\log (m !) \\
\frac{\partial \log (f(t, l, m))}{\partial p}=m / p-\lambda(t-R)+(m-l) /(1-p)=0 \\
\lambda=m /(p(R-t))+(m-l) /((1-p)(R-t)) \\
\hat{p}_{5}=m t /(R(l-m)+m t) \\
\frac{\partial \log (f(t, l))}{\partial \lambda}=1 / \lambda+(l-1) / \lambda-t+t p-p R=0 \\
\lambda=l /(t+p(R-t)) \\
\hat{\lambda}_{6}=[(l-m) / t]+(m / R)
\end{array}
$$


Using $L, M$, derivations of $\hat{p}_{2}$ and $\hat{\lambda}_{2}$ need the assumption of $P(l \mid m)=\left(\begin{array}{c}l-1 \\ m-1\end{array}\right) p^{m-1}(1-p)^{l-m}$. Then, $P(l, m)$ can be written as below and estimators $\hat{p}_{2}$ and $\hat{\lambda}_{2}$ can be derived. Clearly, $\hat{p}_{2}$ can also be obtained by plugging $\hat{\lambda}_{2}$ in $\hat{p}_{2}=\frac{m}{\hat{\lambda}_{2} R}=\frac{m}{l R / R}=\frac{m}{l}$ and $\hat{\lambda}_{2}=\frac{m}{\hat{p}_{2} R}=\frac{m}{m R / l}=\frac{l}{R}$.

$$
\begin{array}{r}
P(l, m)=\left(\begin{array}{c}
l-1 \\
m-1
\end{array}\right) p^{m-1}(1-p)^{l-m} \frac{(\lambda p R)^{m} e^{-\lambda p R}}{(m) !} \\
\log (f(l, m))=m \log (p)+(l-m) \log (1-p)-\lambda p R+m \log (\lambda p R)-\log (m !) \\
\frac{\partial \log (f(l, m))}{\partial p}=m / p-\lambda R+m / p-(l-m) /(1-p)=0 \\
\hat{p}_{2}=m / l \\
\frac{\partial \log (f(m, l))}{\partial \lambda}=m / \lambda-p R=0 \\
\lambda=m /(p R) \\
\lambda=m /((m / l) R) \\
\hat{\lambda}_{2}=l / R
\end{array}
$$

\section{Appendix B. Derivation of Expected Values}

Derivations of expected value of the estimators are presented in this section with approximations mainly utilizing $E(M)=\lambda p R, E(L)=\lambda R-\frac{(1-p)\left(1-e^{\lambda p R}\right)}{p}$, and $E(T)=R-\frac{\left(1-e^{\lambda p R}\right)}{\lambda p}$,

$$
\begin{gathered}
E\left(\hat{\lambda}_{2}\right)=E(l / R)=\frac{E(l)}{R}=\lambda-\frac{(1-p)\left(1-e^{\lambda p R}\right)}{p R} \\
E\left(\hat{\lambda}_{3}\right)=E(l / t)=\int_{0}^{R} \sum_{l=1}^{\infty} \frac{(l) f(t, l)}{t} d t=\sum_{l=1}^{\infty} \int_{0}^{R} \frac{(l)(\lambda p)(\lambda t)^{l-1} e^{-t \theta} e^{-p \lambda R}}{t(l-1) !} d t \\
=\sum_{l=1}^{\infty} \frac{l p(1-p) e^{-\lambda p R}}{(l-1)(1-p)^{l}} \int_{0}^{R} \frac{(\theta)(\theta t)^{l-2} e^{-t \theta} e^{-p \lambda R}}{(l-2) !} d t=\sum_{l=1}^{\infty} \frac{l p(1-p) e^{-\lambda p R}}{(l-1)(1-p)^{l}} \sum_{n=l}^{\infty} \frac{(\theta R)^{n} e^{-\theta R}}{n !} \\
=\theta E\left(\frac{l}{l-1}\right)+\lambda p+\lambda p E(1 / x) \quad \mathrm{x} \sim \operatorname{Poisson}(\lambda R)
\end{gathered}
$$

Approximation to $E\left(\hat{\lambda}_{3}\right)$,

$$
\begin{gathered}
E\left(\hat{\lambda}_{3}\right) \approx E(l / t) \approx \frac{E(l)}{E(t)}=\lambda+\frac{1-e^{\lambda p R}}{R-\frac{1-e^{-\lambda p R}}{\lambda p}} \\
=\lambda+\frac{1}{\lambda R-\frac{\left(1-e^{-\lambda p R}\right)(1-p)}{p}-1}=\frac{\lambda R-\frac{\left(1-e^{-\lambda p R}\right)(1-p)}{p}}{R-\frac{\left(1-e^{-\lambda p R}\right)}{\lambda p}} \\
E\left(\hat{\lambda}_{4}\right)=E((l-1) / t)=\int_{0}^{R} \sum_{1}^{\infty} \frac{(l-1) f(t, l)}{t} d t=\int_{0}^{R} \sum_{l=1}^{\infty} \frac{(l-1)(\lambda p)(\lambda t)^{l-1} e^{-t \theta} e^{-p \lambda R}}{t(l-1) !} d t \\
E\left(\hat{\lambda}_{5}\right)=E(l /((1-p) t+p R)) \approx \frac{\lambda\left(1-e^{-\lambda p R}\right)}{\left(1-e^{-\lambda p R}\right)}=\lambda
\end{gathered}
$$


Since $(l-1) / t$ or $(m-1) / t$ estimators can be evaluated when $L>0$ or $M>0$, then, they need to be normalized by $P(L>0)=P(M>0)=\left(1-e^{-\lambda p R}\right)$.

$$
\begin{array}{r}
E\left(\hat{\lambda}_{6}\right)=E\left(\frac{l-m}{t}+\frac{m}{R}\right)=E\left(\frac{l-1}{t}\right)+E\left(\frac{m-1}{t}\right)+E\left(\frac{m}{R}\right) \\
=\frac{\lambda\left(1-e^{-\lambda p R}\right)-(\lambda p)+\left(\frac{\lambda p R}{R}\right)}{\left(1-e^{-\lambda p R}\right)}=\lambda \\
P(m, l)=\left(\begin{array}{c}
l-1 \\
m-1
\end{array}\right) p^{m-1}(1-p)^{l-m}\left[\frac{p}{(1-p)^{l}} \sum_{n=l}^{\infty} \frac{(\theta R)^{n} e^{-\theta R}}{n !}\right] \\
E(m / l)=\sum_{l=1}^{\infty} \sum_{m=1}^{l-1} \frac{m}{l}\left(\begin{array}{c}
l-1 \\
m-1
\end{array}\right) p^{m-1}(1-p)^{l-m}[P(L=l)] \\
=\sum_{m=1}^{l-1} m\left(\begin{array}{c}
l-1 \\
m-1
\end{array}\right) p^{m-1}(1-p)^{l-m}=p(l-1)+1 \\
=p E\left(\frac{l-1}{l}\right)+E(1 / l)=p+(1-p) E(1 / l)
\end{array}
$$

Approximation to $E\left(\hat{p}_{2}\right)$,

$$
\begin{aligned}
& E(m / l) \approx \frac{E(m)}{E(l)}=\frac{p \lambda p R}{\lambda p R-(1-p)\left(1-e^{-\lambda p R}\right)} \\
& E\left(\hat{p}_{3}\right)=E[1 /(\lambda(R-t))] \approx \frac{p}{\left(1-e^{-\lambda p R}\right)} \\
& E\left(\hat{p}_{4}\right)=E[t /((R-t)(l-1))]=\int_{0}^{R} \sum_{l=2}^{\infty} \frac{t f(t, l)}{(R-t)(l-1)} d t
\end{aligned}
$$

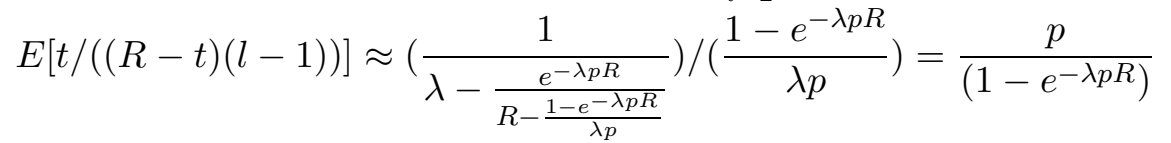

$$
\begin{aligned}
& E(m / t)=\sum_{m=1}^{\infty} \int_{0}^{R} \frac{m f(t, m)}{t} d t=\sum_{m=1}^{\infty} \frac{(m /(m-1)) \lambda p R^{m} e^{-\lambda p R} \lambda p}{(m-1) !} \\
& =\lambda p E[1+1 / k] \quad \text { where } \mathrm{k} \sim \operatorname{Poisson}(\lambda p R) \\
& \approx E(m / t) \approx \frac{E(m)}{E(t)}=\frac{\lambda p R}{R-\frac{1-e^{-\lambda p R}}{\lambda p}} \\
& E((m-1) / t)=\sum_{m=1}^{\infty} \int_{0}^{R} \frac{(m-1) f(t, m)}{t} d t=\sum_{m=1}^{\infty} \frac{((m-1) /(m-1)) \lambda p R^{m} e^{-\lambda p R} \lambda p}{(m-1) !}=\lambda p \\
& E(m t)=\sum_{m=1}^{\infty} \int_{0}^{R} m t f(t, m) d t=\int_{0}^{R}(\lambda p t)^{2}+\lambda p t d t=\frac{\lambda p R^{2}(2 \lambda p R+3)}{6} \\
& E\left(\hat{p}_{5}\right)=E[m t /(m t+(l-m) R)]=\int_{0}^{R} \sum_{l=1}^{\infty} \sum_{m=1}^{l-1} \frac{m t f(t, l, m)}{m t+(l-m) R} d t
\end{aligned}
$$

Approximation to $E\left(\hat{p}_{5}\right)$,

$$
\begin{aligned}
E[m t /(m t & +(l-m) R)] \approx \frac{\frac{\lambda p R^{2}(2 \lambda p R+3)}{6}}{\frac{\lambda p R^{2}(2 \lambda p R+3)}{6}+\lambda R^{2}-\frac{R(1-p)\left(1-e^{\lambda p R}\right)}{p}-\lambda p R^{2}} \\
& =\frac{E(m)}{E(m)+R E(l-1 / t)-R E(m-1 / t)}=\frac{\lambda p R}{\lambda p+\lambda R-\lambda p}=p
\end{aligned}
$$




\section{Appendix C. Derivation of Estimator Errors}

Derivations of the estimation errors are given between Eqns. (C.2)-(C.12) where $d(P)$ is estimator function.

$$
\begin{aligned}
& E_{\lambda}(d(P)-\lambda)^{2}=V_{\lambda}(d(P))+\left[E_{\lambda}(d(P))-\lambda\right]^{2}=V_{\lambda}(d(P))+\left[\text { Bias }_{\lambda} d(P)\right]^{2} \\
& E\left(\hat{\lambda}_{1}-\lambda\right)^{2}=E(m / p R-\lambda)^{2}=\left(E(m-\lambda p R)^{2}\right) /(p R)^{2}=\lambda / p R \\
& E\left(\hat{\lambda}_{2}-\lambda\right)^{2}=E(l / R-\lambda)^{2}=\frac{E\left(l^{2}\right)}{R^{2}}-\frac{2 \lambda E(l)}{R}+\lambda^{2} \\
& =\lambda^{2}+\frac{3 \lambda p-2 \lambda}{R p}+\frac{\left(1-e^{\lambda p R}\right)\left(p^{2}-3 p+2+2 \lambda(1-p)\right)}{R^{2} p^{2}}-\frac{2 \lambda}{R}\left[\lambda R-\frac{(1-p)\left(1-e^{\lambda p R}\right)}{p}\right]+\lambda^{2} \\
& =\frac{\lambda(3 p-2)}{R p}+\frac{\left(1-e^{\lambda p R}\right)\left(p^{2}-3 p+2+2 \lambda(1-p)\right)}{R^{2} p^{2}} \\
& E\left(\hat{\lambda}_{3}-\lambda\right)^{2}=E(l / t-\lambda)^{2}=E\left[(l / t)^{2}\right]-2 \lambda E(l / t)+\lambda^{2} \\
& =\int_{0}^{R} \sum_{l=1}^{\infty} \frac{\left(l^{2}\right) f(t, l)}{t^{2}} d t-2 \lambda \int_{0}^{R} \sum_{1}^{\infty} \frac{(l) f(t, l)}{t} d t+\lambda^{2} \\
& E\left(\hat{\lambda}_{4}-\lambda\right)^{2}=E_{\lambda}([(l-1) / t]-\lambda)^{2}=E\left[((l-1) / t)^{2}\right]-2 \lambda E((l-1) / t)+\lambda^{2} \\
& =\sum_{l=1}^{\infty} \frac{(l-1) p \theta^{2} R e^{-p \lambda R}}{(l-2)(1-p)^{l}} \sum_{n=l-2}^{\infty} \frac{(\theta R)^{n} e^{-\theta R}}{n !}-2 \lambda\left(1-e^{-\lambda p R}\right)+\lambda^{2} \\
& \left.E\left(\hat{\lambda}_{5}-\lambda\right)^{2}=E_{\lambda}(l /(t+p(R-t))]-\lambda\right)^{2} \\
& \left.=E_{\lambda}(l /(t+p(R-t))-\lambda)^{2}=\int_{0}^{R} \sum_{l=1}^{\infty}(l /(t+p(R-t))]-\lambda\right)^{2} f(t, l) d t \\
& E\left(\hat{\lambda}_{6}-\lambda\right)^{2}=E_{\lambda}([(l-m) / t+R / m]-\lambda)^{2} \\
& E_{\lambda}([(l-m) / t+R / m]-\lambda)^{2}=\int_{0}^{R} \sum_{l=1}^{\infty} \sum_{m=1}^{l-1}([(l-m) / t+R / m]-\lambda)^{2} f(t, l, m) d t
\end{aligned}
$$

For unknown $p$ case,

$$
\begin{gathered}
E\left(\hat{p}_{1}-p\right)^{2}=E_{p}(m / \lambda R-p)^{2}=p /(\lambda R) \\
E\left(\hat{p}_{2}-p\right)^{2}=E_{p}(m / l-p)^{2}=E\left[(m / l)^{2}\right]-2 p E(m / l)+p^{2} \\
=E\left[\frac{(l-1) p(l p-2 p+3)}{l^{2}}\right]-2 p(p+(1-p) E(1 / l))+p^{2} \\
E\left(\hat{p}_{3}-p\right)^{2}=E_{p}(1 /[\lambda(R-t)]-p)^{2}=E_{p}(1 /[\lambda(R-t)]-p)^{2} \\
=\int_{0}^{R} \sum_{l=1}^{\infty}(1 /[\lambda(R-t)]-p)^{2} f(t, l) d t \\
E\left(\hat{p}_{4}-p\right)^{2}=E_{p}(t /[(l-1)(R-t)]-p)^{2} \\
=\int_{0}^{R} \sum_{l=1}^{\infty}(t /[(l-1)(R-t)]-p)^{2} f(t, l) d t \\
E\left(\hat{p}_{5}-p\right)^{2}=E_{p}(m t /[m t+(l-m) R]-p)^{2} \\
=\int_{0}^{R} \sum_{l=1}^{\infty} \sum_{m=1}^{l-1}(m t /[m t+(l-m) R]-p)^{2} f(t, l, m) d t
\end{gathered}
$$




\section{Appendix C.1. Derivation of Analytical Error Bounds}

Analytical estimation error bounds for single cycle probe information can be found by utilizing the Cramér-Rao Lower Bound under the assumptions of Poisson arrivals and $Q=0$.

$$
\begin{aligned}
& \frac{\partial \log ^{2}(f(t, l))}{\partial p^{2}}=-1 / p^{2} \\
& V(\hat{p}) \geq \frac{1}{-n E\left(-1 / p^{2}\right)}=\frac{p^{2}}{n} \\
& \frac{\partial \log ^{2}(f(t, l))}{\partial \lambda^{2}}=-l / \lambda^{2} \\
& V(\hat{\lambda}) \geq \frac{1}{-n E\left(-l / \lambda^{2}\right)}=\frac{\lambda^{2}}{n E(l)} \\
& \frac{\partial \log ^{2}(f(l, m))}{\partial p^{2}}=\left[-2 m / p^{2}\right]+\left[-(l-m) /(1-p)^{2}\right] \\
& V(\hat{p}) \geq \frac{1}{-n E\left(\left[-2 m / p^{2}\right]+\left[-(l-m) /(1-p)^{2}\right]\right)}=\frac{1}{n E\left(\left[2 m / p^{2}\right]+\left[(l-m) /(1-p)^{2}\right]\right)} \\
& \frac{\partial \log ^{2}(f(m, l))}{\partial \lambda^{2}}=-m / \lambda^{2} \\
& V(\hat{\lambda}) \geq \frac{1}{-n E\left(\left[-m / \lambda^{2}\right]\right)}=\frac{\lambda}{n p R} \\
& \frac{\partial \log ^{2}(f(t, l, m))}{\partial p^{2}}=\left[-m / p^{2}\right]+\left[-(l-m) /(1-p)^{2}\right] \\
& V(\hat{p}) \geq \frac{1}{-n E\left(\left[-m / p^{2}\right]+\left[-(l-m) /(1-p)^{2}\right]\right)}=\frac{1}{n E\left(\left[m / p^{2}\right]+\left[(l-m) /(1-p)^{2}\right]\right)} \\
& \frac{\partial \log (f(t, l, m))}{\partial \lambda^{2}}=-l / \lambda^{2} \\
& V(\hat{\lambda}) \geq \frac{1}{-n E\left(-l / \lambda^{2}\right)}=\frac{\lambda^{2}}{n E(l)}
\end{aligned}
$$

\section{Appendix D. Queue Length Estimators}

For $Q=0$, queue length estimation models with combination of different parameter estimators can be simply calculated by following formulae. Similarly for $Q \geq 0, \hat{p}, \hat{\lambda}$ combinations can be inputted into Eqs.(13) and (17).

$$
\begin{gathered}
E\left(N \mid l, t, m, Q_{i}=0\right)=l+(l-m / l)(l / R)(R-t)=l+(l-m)(1-t / R) \\
E\left(N \mid l, t, m, Q_{i}=0\right)=l+(1-m / l)[(l) / t](R-t)=m+\frac{R(l-m)}{t} \\
E\left(N \mid l, t, m, Q_{i}=0\right)=l+(1-m / l)[(l-1) / t](R-t) \\
E\left(N \mid l, t, m, Q_{i}=0\right)=l+(1-m / l)((l-m) / t+m / R)(R-t)=m+\frac{R(l-m)}{t} \\
E\left(N \mid l, t, m, Q_{i}=0\right)=l+\left(1-\frac{m t}{m t+(l-m) R}\right)(l / R)(R-t) \\
E\left(N \mid l, t, m, Q_{i}=0\right)=l+\left(1-\frac{m t}{m t+(l-m) R}\right)(l / t)(R-t) \\
E\left(N \mid l, t, m, Q_{i}=0\right)=l+\left(1-\frac{m t}{m t+(l-m) R}\right)((l-1) / t)(R-t)
\end{gathered}
$$




$$
E\left(N \mid l, t, m, Q_{i}=0\right)=l+\left(1-\frac{m t}{m t+(l-m) R}\right)((l-m) / t+m / R)(R-t)=m+\frac{R(l-m)}{t}
$$

\section{Appendix E. Set Up for Simulations}

The VISSIM simulation set up in this paper is adopted from Comert (2013b). Queue length estimation models are revised and rerun since cycle-to-cycle overflow queue formulations are used in this paper. VISSIM generates vehicles with exponential interarrival times at the origin that move and queue realistically. The arrival profile changes as vehicles move along the network based on vehicle composition, vehicle characteristics, driving behavior, number of lanes, and other network settings. Similar to the complex real-life traffic systems with multiple parameters to control, detailed analysis could be done in order to make sure a fully accurate comparison under different scenarios which is beyond the scope of this paper. For a basic evaluation purpose a single lane fixed time controlled isolated intersection located 4,921 ft downstream of a 9,797 ft link. The approach length is tested for having enough capacity to hold

long queues. Fixed time signal with Red $=45$ seconds (s), Green=45 s, Cycle Length $=90 \mathrm{~s}$, and no amber time is programmed.

Saturation flow rate for the designed intersection is observed to be around 1,000 vehicles per hour (vph) which showed approximately the saturation headway of $1.80 \mathrm{spv}$. It is recorded that approximately 24 vehicles could be discharged within a cycle yielding to Effective Green $\approx 1.8 * 24=43.2$ s. Based on these observations, 600,700, 800, 900, and 985 vph volumes yielding approximately $\rho=0.60,0.70,0.80$, 0.88, and 0.98 are used respectively in VISSIM and analytical model comparisons. VISSIM is run 1,000 cycles for 4 different random seeds (121-124) and 12 probe percentages at each demand level. First 50 cycles of the runs are not included in estimation error calculations for warm up period. Queue definition in terms of min, max speed, and max queue length is given as follows; for arrival rates 600, 700, 800, and $900 \mathrm{vph}(<6.2 \mathrm{mph},>9.3 \mathrm{mph}$, and 1,640.5 ft); and for close to saturated flow $985 \mathrm{vph}(<9.3$ $\mathrm{mph},>12.4 \mathrm{mph}$, and 3,281 ft) are used. Notice that for high volumes, in queue is not defined as strict as the low volumes since vehicles stop and go at very low speeds near saturation flow level. In point queue simulations for Eqs. (13) and (17), vehicles are discharged at a constant $1.80 \mathrm{spv}$ for five different arrival rates of $\lambda=0.163,0.190,0.218,0.239$, and 0.267 vehicle per second (vps) yielding volume-to-capacity ratios of $\rho=0.60,0.70,0.80,0.88$, and 0.98 respectively. They are calculated by dividing $\lambda$ to the maximum departure rate during effective green interval, which is equal to $0.272 \mathrm{vps}$ (i.e., 24 vehicles $/ 88.20 \mathrm{~s}=0.272$ vps). Point queue simulation is run for 51,000 cycles for each probe proportion and volume level. Using same $\rho$ values, analytical estimation models are evaluated in VISSIM. 


\section{References}

Adams, W. F., 1936. Road traffic considered as a random series. Institution of Civil Engineers Journal 4 (1), 121-130.

Akçelik, R., 1980. Time-dependent expressions for delay, stop rate and queue length at traffic signals. Australian Road Research Board Melbourne, Australia.

Anderson, L. A., Canepa, E. S., Horowitz, R., Claudel, C. G., Bayen, A., 2014. Optimization-based queue estimation on an arterial traffic link with measurement uncertainties. In: Transportation Research Board 93rd Annual Meeting. No. 14-4570.

Ban, X. J., Hao, P., Sun, Z., 2011. Real time queue length estimation for signalized intersections using travel times from mobile sensors. Transportation Research Part C: Emerging Technologies 19 (6), $1133-1156$.

Boon, M., Adan, I., Winands, E., Down, D., 2012. Delays at signalized intersections with exhaustive traffic control. Probability in the Engineering and Informational Sciences 26 (03), 337-373.

Bowei, X., Michailidis, G., Nair, V. N., 2006. Estimating network loss rates using active tomography. Journal of the American Statistical Association 101 (476), 1430-1448.

Boyce, D. E., Hicks, J., Sen, A., 1991. In-vehicle navigation requirements for monitoring link travel times in a dynamic route guidance system. Operations Review 8 (1), 17-24.

Buckley, D., 1962. Road traffic headway distributions. In: Australian Road Research Board (ARRB) Conference, 1st, Canberra. Vol. 1.

Calabrese, F., Colonna, M., Lovisolo, P., Parata, D., Ratti, C., 2011. Real-time urban monitoring using cell phones: A case study in rome. IEEE Transactions on Intelligent Transportation Systems 12 (1), $141-151$.

Cantieni, G., Ni, Q., Barakat, C., Turletti, T., 2005. Performance analysis under finite load and improvements for multirate 802.11. Computer Communications 28 (10), 1095-1109.

Cetin, M., List, G. F., Zhou, Y., 2005. Factors affecting the minimum number of probes required for reliable travel time estimation. Transportation Research Record 1917, 37-44.

Chen, M., Chien, S. I. J., 2000. Determining the number of probe vehicles for freeway travel time prediction by microscopic simulation. Transportation Research Record 1719, 61-68.

Cheng, Y., Qin, X., Jin, J., Ran, B., Anderson, J., 2011. Cycle-by-cycle queue length estimation for signalized intersections using sampled trajectory data. Transportation Research Record: Journal of the Transportation Research Board 2257 (1), 87-94.

Cheu, R. L., Xie, C., Lee, D., 2002. Probe vehicle population and sample size for arterial speed prediction. Computer-Aided Civil and Infrastructure Engineering 17 (1), 53-60.

Coifman, B., Kim, S., 2009. Speed estimation and length based vehicle classification from freeway singleloop detectors. Transportation Research Part C: Emerging Technologies 17 (4), 349-364.

Comert, G., 2013a. Effect of stop line detection in queue length estimation at traffic signals from probe vehicles data. European Journal of Operational Research 226 (1), 67-76.

Comert, G., 2013b. Simple analytical models for estimating the queue lengths from probe vehicles at traffic signals. Transportation Research Part B: Methodological 55, 59-74. 
Comert, G., Cetin, M., 2009. Queue length prediction from probe vehicle location and the impacts of sample size. European Journal of Operational Research 197 (1), 196-202.

Comert, G., Cetin, M., 2011. Analytical evaluation of the error in queue length estimation at traffic signals from probe vehicle data. IEEE Transactions on Intelligent Transportation Systems 12 (2), 563-573.

Comert, G., Cetin, M., Nichols, E., 2009. Incorporating queue length measurements into actuated signal control: Evaluation of efficiency benefits at an intersection. In: TRB 88th Annual Meeting Compendium of Papers DVD. Vol. 1. Washington, D.C.

Dailey, D. J., 1999. A statistical algorithm for estimating speed from single loop volume and occupancy measurements. Transportation Research Part B 33 (5), 313-322.

Darroch, J. N., 1964. On the traffic-light queue. The Annals of Mathematical Statistics 35 (1), 380-388.

Dion, F., Oh, J.-S., Robinson, R., 2011. Virtual testbed for assessing probe vehicle data in intellidrive systems. IEEE Transactions on Intelligent Transportation Systems 12 (3), 635-644.

Du, J., Barth, M. J., 2008. Next-generation automated vehicle location systems: positioning at the lane level. IEEE Transactions on Intelligent Transportation Systems 9 (1), 48-57.

Duffield, N. G., 2006. Network tomography of binary network performance characteristics. IEEE Transactions on Information Theory 52 (12), 5373-5388.

Feng, Y., Head, K. L., Khoshmagham, S., Zamanipour, M., 2015. A real-time adaptive signal control in a connected vehicle environment. Transportation Research Part C: Emerging Technologies.

Feng, Y., Hourdos, J., Davis, G. A., 2014. Probe vehicle based real-time traffic monitoring on urban roadways. Transportation Research Part C: Emerging Technologies 40, 160-178.

Ferman, M. A., Blumenfeld, D. E., Dai, X., 2005. An analytical evaluation of a real-time traffic information system using probe vehicles. Journal of Intelligent Transportation Systems 9 (1), 23-24.

Fu, L., Hellinga, B., 2000. Delay variability at signalized intersections. Transportation Research Record $1710,215-221$.

Gao, H., Liu, F., 2013. Estimating freeway traffic measures from mobile phone location data. European Journal of Operational Research 229 (1), 252-260.

Garetto, M., Chiasserini, C.-F., 2005. Performance analysis of 802.11 wlans under sporadic traffic. In: NETWORKING 2005. Networking Technologies, Services, and Protocols; Performance of Computer and Communication Networks; Mobile and Wireless Communications Systems. Springer, pp. 13431347.

Gartner, N. H., Pooran, F. J., Andrews, C. M., 2002. Optimized policies for adaptive control systems: implementation and field testing. Transportation Research Record 1811, 148-156.

Goodall, N. J., Park, B., Smith, B. L., 2014. Microscopic estimation of arterial vehicle positions in a low-penetration-rate connected vehicle environment. Journal of Transportation Engineering 140 (10).

Goodall, N. J., Smith, B. L., Park, B. B., 2013. Traffic signal control with connected vehicles. Transportation Research Record: Journal of the Transportation Research Board 2381 (1), 65-72.

Haight, F. A., 1959. Overflow at a traffic flow. Biometrika 46 (3-4), 420-424.

Hao, P., Ban, X. J., Guo, D., Ji, Q., 2014. Cycle-by-cycle intersection queue length distribution estimation using sample travel times. Transportation research part B: methodological 68, 185-204. 
Hao, P., Sun, Z., Ban, X. J., Guo, D., Ji, Q., 2013. Vehicle index estimation for signalized intersections using sample travel times. Transportation Research Part C: Emerging Technologies 36, 513-529.

Hartenstein, H., Laberteaux, K. P., 2008. A tutorial survey on vehicular ad hoc networks. Communications Magazine, IEEE 46 (6), 164-171.

Heidemann, D., 1994. Queue length and delay distributions at signalized intersections. Transportation Research Part B 28 (5), 624-635.

Hellinga, B., Fu, L., 1999. Assessing expected accuracy of probe vehicle travel time reports. Journal of Transportation Engineering 125 (6), 524-530.

Hellinga, B. R., 2002. Improving freeway speed estimates from single-loop detectors. Journal of Transportation Engineering 128 (1), 58-67.

Hellinga, B. R., Fu, L., 2002. Reducing bias in probe-based arterial link travel time estimates. Transportation Research Part C: Emerging Technologies 10 (4), 257-273.

Herrera, J. C., Bayen, A. M., 2010. Incorporation of lagrangian measurements in freeway traffic state estimation. Transportation Research Part B 44 (4), 460-481.

Hofleitner, A., Herring, R., Bayen, A., 2012. Arterial travel time forecast with streaming data: A hybrid approach of flow modeling and machine learning. Transportation Research Part B 46 (9), 1097-1122.

Jenelius, E., Koutsopoulos, H. N., 2013. Travel time estimation for urban road networks using low frequency probe vehicle data. Transportation Research Part B 53, 64-81.

Jordan, C., Cetin, M., 2014. Improving travel times for emergency response vehicles: Traffic control strategies based on connected vehicles technologies. Tech. rep.

Kang, Y. S., 2000. Delay, stop and queue estimation for uniform and random traffic arrivals at fixed-time signalized intersections. Ph.D. thesis, Virginia Polytechnic Institute and State University, Blacksburg, Virginia.

Kianfar, J., Edara, P., 2010. Optimizing freeway traffic sensor locations by clustering global-positioningsystem-derived speed patterns. IEEE Transactions on Intelligent Transportation Systems 11 (2), 738747.

Kwella, B., Lehmann, H., 2000. Floating car data analysis of urban road networks. Lecture Notes in Computer Science 1798, 357-367.

Lee, S., Wong, S., Li, Y., 2015. Real-time estimation of lane-based queue lengths at isolated signalized junctions. Transportation Research Part C: Emerging Technologies 56, 1-17.

Li, B., 2010. Bayesian inference for vehicle speed and vehicle length using dual-loop detector data. Transportation Research Part B 44 (1), 108-119.

Lin, I., Rong, H., Kornhauser, A. L., 2008. Estimating nationwide link speed distribution using probe position data. Journal of Intelligent Transportation Systems 12 (1), 29-37.

Liu, H., Ma, W., 2009. A virtual vehicle probe model for time-dependent travel time estimation on signalized arterials. Transportation Research Part C: Emerging Technologies 17 (1), 11-26.

Lu, Y., Yang, X., 2014. Estimating dynamic queue distribution in a signalized network through a probability generating model. Intelligent Transportation Systems, IEEE Transactions on 15 (1), 334-344. 
McNeil, D. R., 1968. A solution to the fixed cycle traffic light problem for compound poisson arrivals. Journal of Applied Probability 5 (3), 624-635.

Medhi, J., 1991. Stochastic models in queuing theory. Academic Press, Inc. Harcourt Brace Jovanovich Publishers.

Miller, A., 1963. Settings for fixed-cycle traffic signals. Operations Research Quarterly 14, 373-386.

Mirchandani, P. B., Zou, N., 2007. Queuing models for analysis of traffic adaptive signal control. IEEE Transactions on Intelligent Transportation Systems 8 (1), 50-59.

Newell, G. F., 1965. Approximation methods for queues with application to the fixed-cycle traffic light. SIAM Review 7 (2), 223-240.

Newell, G. F., 1982. Applications of queueing theory, 2nd Edition.

Ohno, K., 1978. Computational algorithm for a fixed cycle traffic signal and new approximate expressions for average delay. Transportation Science 12 (1), 29-47.

Olszewski, P., 1990. Modeling of queue probability distribution at traffic signals. In: M.Koshi (Ed.), Transportation and Traffic Theory. Elsevier, Amsterdam.

Ou, Q., Bertini, R. L., van Lint, J., Hoogendoorn, S., 2011. A theoretical framework for traffic speed estimation by fusing low-resolution probe vehicle data. IEEE Transactions on Intelligent Transportation Systems 12 (3), 747-756.

Park, H., Miloslavov, A., Lee, J., Veeraraghavan, M., Park, B., Smith, B. L., 2011. Integrated trafficcommunication simulation evaluation environment for intellidrive applications using sae j2735 message sets. Transportation Research Record: Journal of the Transportation Research Board 2243 (1), 117126.

Patire, A. D., Wright, M., Prodhomme, B., Bayen, A., 2015. How much gps data do we need? Transportation Research Part C: Emerging Technologies 58 (B), 325-342.

Paxson, V., Floyd, S., 1995. Wide area traffic: the failure of poisson modeling. IEEE/ACM Transactions on Networking 3 (3), 226-244.

Qing, O., Bertini, L. R., Lint, J. W. C. V., Hoogendoorn, S. P., 2011. A theoretical framework for traffic speed estimation by fusing low-resolution probe vehicle data. IEEE Transactions on Intelligent Transportation Systems 12 (3), 747-756.

Quiroga, C. A., Bullock, D., 1998. Travel time studies with global positioning and geographic information systems: an integrated methodology. Transportation Research Part C: Emerging Technologies 6 (1), $101-127$.

Ramezani, M., Geroliminis, N., 2012. On the estimation of arterial route travel time distribution with markov chains. Transportation Research Part B 46 (10), 1576-1590.

Ramezani, M., Geroliminis, N., 2015. Queue profile estimation in congested urban networks with probe data. Computer-Aided Civil and Infrastructure Engineering 30 (6), 414-432.

Rouphail, N., Tarko, A., Li, J., 2001. Traffic flows at signalized intersections. In: Traffic Flow Theory Monograph.

Seo, T., Kusakabe, T., Asakura, Y., 2015. Estimation of flow and density using probe vehicles with spacing measurement equipment. Transportation Research Part C: Emerging Technologies 53, 134-150. 
Smith, B. L., Venkatanarayana, R., Park, H., Goodall, N., Datesh, J., Skerrit, C., 2010. Intellidrivesm traffic signal control algorithms. University of Virginia.

Sohn, K., Hwang, K., 2008. Space-based passing time estimation on a freeway using cell phones as traffic probes. IEEE Transactions on Intelligent Transportation Systems 9 (3), 559-568.

Srinivasan, K. K., Jovanis, P. P., 1996. Determination of number of probe vehicles required for reliable travel time measurement in urban network. Transportation Research Record 1537, 5-22.

Su, P., Lee, J., Park, B. B., 2014. Calibrating communication simulator for connected vehicle applications. Journal of Intelligent Transportation Systems (ahead-of-print), 1-11.

Sun, Z., Ban, X. J., 2013. Vehicle classification using gps data. Transportation Research Part C: Emerging Technologies 37, 102-117.

Tarko, A., Rouphail, N., 1994. Distribution-free model for estimating random queues in signalized networks. Transportation Research Record 1457, 192-197.

Tiaprasert, K., Zhang, Y., Wang, X. B., Zeng, X., 2015. Queue length estimation using connected vehicle technology for adaptive signal control. IEEE Transactions on Intelligent Transportation Systems 16, 2129-2140.

Turner, S., Holdener, D., 1995. Probe vehicle sample sizes for real-time information: the houston experience. In: Proceedings of the Vehicle Navigation and Information Systems Conference. Seattle, WA.

Van Den Broek, M., Van Leeuwaarden, J., Adan, I., Boxma, O., 2006. Bounds and approximations for the fixed-cycle traffic-light queue. Transportation science 40 (4), 484-496.

Van Leeuwaarden, J., 2006. Delay analysis for the fixed-cycle traffic-light queue. Transportation Science 40 (2), 189-199.

Viti, F., 2006. The dynamics and the uncertainty of delays at signals. Ph.D. thesis, Delft University of Technology, Delft, the Netherlands.

Viti, F., Van Zuylen, H., 2010. Probabilistic models for queues at fixed control signals. Transportation Research Part B 44 (1), 120-135.

Webster, F. V., 1958. Traffic signal settings. road research technical paper no. 39. Road Research Laboratory.

Zheng, F., 2011. Modeling urban travel times. Ph.D. thesis, Delft University of Technology, Delft, the Netherlands. 